\title{
Unlimited Damage Accumulation in Metallic Materials Under Cascade- Damage Conditions
}

September 2008

\author{
A. V. BARASHEV \\ The University of Liverpool, UK \\ S. I. GOLUBOV \\ Oak Ridge National Laboratory, \\ University of Tennessee
}




\title{
DOCUMENT AVAILABILITY
}

Reports produced after January 1, 1996, are generally available free via the U.S. Department of Energy (DOE) Information Bridge.

\section{Web site http://www.osti.gov/bridge}

Reports produced before January 1, 1996, may be purchased by members of the public from the following source.

\author{
National Technical Information Service \\ 5285 Port Royal Road \\ Springfield, VA 22161 \\ Telephone 703-605-6000 (1-800-553-6847) \\ TDD 703-487-4639 \\ Fax 703-605-6900 \\ E-mail info@ntis.gov \\ Web site http://www.ntis.gov/support/ordernowabout.htm
}

Reports are available to DOE employees, DOE contractors, Energy Technology Data Exchange (ETDE)

representatives, and International Nuclear Information System (INIS) representatives from the following source.

Office of Scientific and Technical Information

P.O. Box 62

Oak Ridge, TN 37831

Telephone 865-576-8401

Fax 865-576-5728

E-mail reports@osti.gov

Web site http://www.osti.gov/contact.html

This report was prepared as an account of work sponsored by an agency of the United States Government. Neither the United States Government nor any agency thereof, nor any of their employees, makes any warranty, express or implied, or assumes any legal liability or responsibility for the accuracy, completeness, or usefulness of any information, apparatus, product, or process disclosed, or represents that its use would not infringe privately owned rights. Reference herein to any specific commercial product, process, or service by trade name, trademark, manufacturer, or otherwise, does not necessarily constitute or imply its endorsement, recommendation, or favoring by the United States Government or any agency thereof. The views and opinions of authors expressed herein do not necessarily state or reflect those of the United States Government or any agency thereof. 
Materials Science and Technology Division

UNLIMITED DAMAGE ACCUMULATION IN METALLIC MATERIALS UNDER CASCADE-DAMAGE CONDITIONS

A. V. BARASHEV

The University of Liverpool, UK

S. I. GOLUBOV

Oak Ridge National Laboratory,

University of Tennessee

September 2008

Prepared by

OAK RIDGE NATIONAL LABORATORY

Oak Ridge, Tennessee 37831-6283

managed by

UT-BATTELLE, LLC

for the

U.S. DEPARTMENT OF ENERGY

under contract DE-AC05-00OR22725 


\section{CONTENTS}

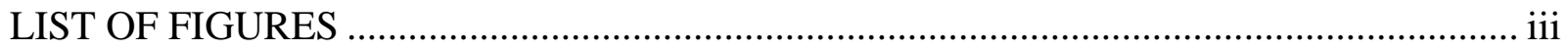

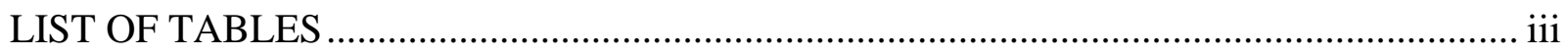

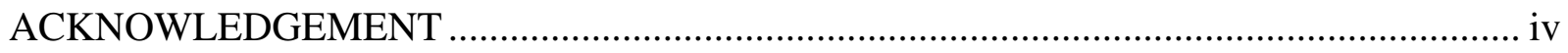

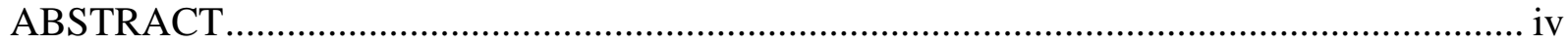

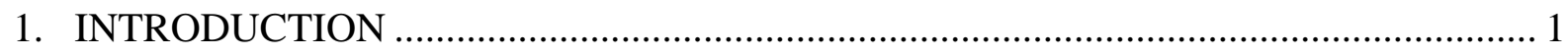

2. GROWTH AND SATURATION OF VOIDS DISTRIBUTED RANDOMLY ............... 4

3. ONSET OF SPATIAL CORRELATIONS DUE TO 1-3 MIGRATING SIA

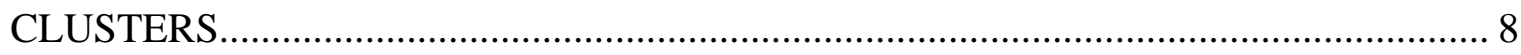

4. EFFECT OF CORRELATIONS ON SWELLING RATE .......................................... 11

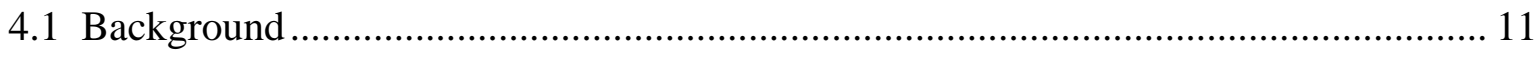

4.2 Void swelling in the absent of repulsive barriers............................................. 12

4.3. Void swelling in the presence of repulsive barriers not correlated with voids ............ 12

4.4. Void swelling in the presence of correlations .................................................. 13

4.5. Calculation of correlation-screening factors ................................................... 15

4.6. Interpretation of the universal swelling rate observed in stainless steels .................. 16

5. INFLUENCE OF SOLUTE ATMOSPHERES ...................................................... 17

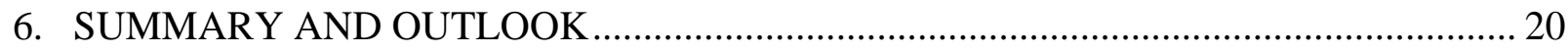

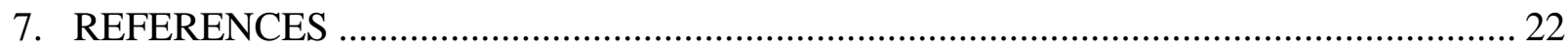

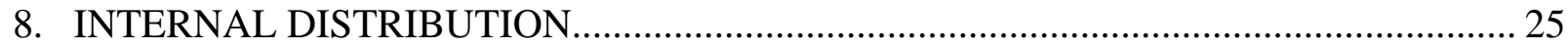

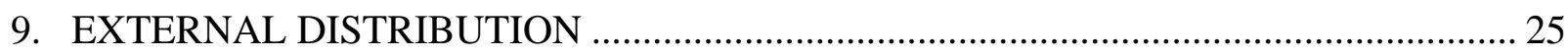




\section{LIST OF FIGURES}

Figure

1. The dependence of the saturated void radius on the ratio of the dislocation bias and the fraction of SIAs produced in the form of 1-D migrating SIA

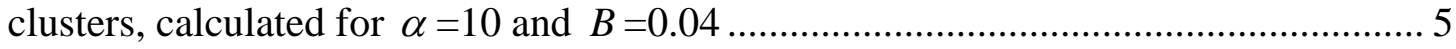

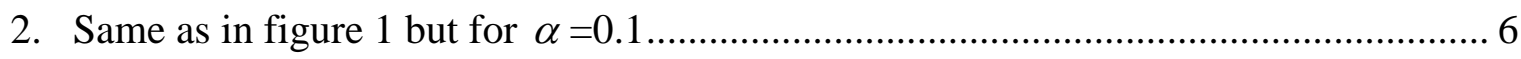

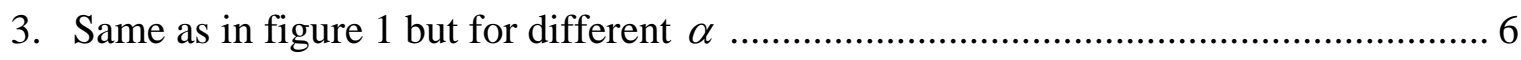

4. Dependence of swelling on irradiation dose calculated using equation (17) for $N=10^{22} \mathrm{~m}^{-3}, r_{\mathrm{m}}=5 \mathrm{~nm}, \varepsilon_{\mathrm{i}}^{\mathrm{g}}=0.2, \eta_{\text {bar }} \sigma_{\text {bar }} / \sigma_{\mathrm{d}}=1, \varepsilon_{\text {surv }}=0.1$ and different values of the correlation-screening factor of voids, $\eta_{\mathrm{v}}$. The curve with full squares has been calculated for correlations developing with irradiation dose (see text).

5. Concentration profiles of a component $\mathrm{A}$ in a binary $\mathrm{AB}$ alloy, $C_{\mathrm{A}}$, normalized by the initial concentration $C_{\mathrm{A} 0}$, calculated near a plain grain boundary for different velocities of the boundary motion. The boundary moves from the left to the right

\section{LIST OF TABLES}

Table

1. Determination of probabilities of different reactions for 1-D mobile SIA clusters in a system containing voids, dislocations and repulsive barriers (subscript 'bar') 


\section{ACKNOWLEDGEMENTS}

The authors express their gratitude to Dr B. N. Singh (Risø National Laboratory, Denmark), Dr Helmut Trinkaus (Forschungscentrum Jülich, Germany), Dr S. J. Zinkle (Oak Ridge National Laboratory, USA) and Prof D. J. Bacon (The University of Liverpool, UK) for careful reading and useful discussions of the manuscript. The research was sponsored by a research grant from the UK Engineering and the Physical Sciences Research Council and the EU-funded project FP 7 GETMAT (A.V.B) and by the Office of Fusion Energy Sciences U.S. Department of Energy, under contract DE-AC05-00OR22725 with UTBattelle, LLC (S.I.G.). 


\begin{abstract}
Most experiments on neutron or heavy-ion cascade-produced irradiation of pure metals and metallic alloys demonstrate unlimited void growth as well as development of the dislocation structure. In contrast, the theory of radiation damage predicts saturation of void swelling at sufficiently high irradiation doses and, accordingly, termination of accumulation of interstitial-type defects. It is shown in the present paper that, under conditions of steady production of one-dimensionally (1-D) mobile clusters of selfinterstitial atoms (SIAs) in displacement cascades, any one of the following three conditions can result in indefinite damage accumulation. First, if the fraction of SIAs generated in the clustered form is smaller than some finite value of the order of the dislocation bias factor. Second, if solute, impurity or transmuted atoms form atmospheres around voids and repel the SIA clusters. Third, if spatial correlations between voids and other defects, such as second-phase precipitates and dislocations, exist that provide shadowing of voids from the SIA clusters. The driving force for the development of such correlations is the same as for void lattice formation and is argued to be always present under cascade-damage conditions. It is emphasised that the mean-free path of 1-D migrating SIA clusters is typically at least an order of magnitude longer than the average distance between microstructural defects; hence spatial correlations on the same scale should be taken into consideration. A way of developing a predictive theory is discussed. An interpretation of the steady-state swelling rate of $\sim 1 \% /$ dpa observed in austenitic steels is proposed
\end{abstract}




\section{INTRODUCTION}

Efforts of many scientists for more than a half of a century have resulted in substantial understanding of the response of various materials to irradiation. A spectrum of new radiation-induced phenomena emerged: void swelling, hardening, creep, growth, formation of ordered structures, modification of phase diagrams, etc. The contribution of theory to understanding of the radiation-induced processes was significant. For example, the development of the NRT standard for a common measure of the irradiation dose in different materials [1], the theory of homogeneous nucleation of point defect clusters [2,3], the Standard Rate Theory (SRT) of void swelling ([4-9]) and its further development for the inclusion of vacancy clustering in cascades, the BEK model [10], all these constituted and complemented each other in theory of radiation damage. Two phenomena were predicted before their observation: void swelling by Greenwood, Foreman and Rimmer in 1959 [11] (discovered by Cawthorne and Fulton in 1966 [12,13]) and radiation-induced segregation (RIS) by Anthony in 1972 [14] (first observed by Okamoto, Harkness and Laidler in 1973 [15]). Also, Foreman [16] predicted in 1972 the existence of onedimensional transport of interstitial-type defects along close-packed crystallographic directions in metals under neutron irradiation to explain the void lattice formation, which was first observed by Evans in molybdenum in 1971 [17].

From a critical point of view, however, the existing information was not understood to a level sufficient to provide the theory with a leading role in creating radiation-resistant materials. Moreover, some observations were in contradiction with the SRT and BEK model. These include higher swelling rates near grain boundaries than in the grain interior in the following cases: high purity copper and aluminium irradiated with fission neutrons or $600 \mathrm{MeV}$ protons (see original references in reviews [18,19]); aluminium irradiated with $225 \mathrm{MeV}$ electrons [20]; neutron-irradiated nickel [21] and stainless steel [22]. Furthermore, the swelling rate at very low dislocation density in copper is higher [23-25], and the dependence of the swelling rate on the densities of voids and dislocations is different [26], than predicted by the SRT. It gradually became clear that something important was missing in the theory. There was evidence that this missing part could not be all the effect of solute and impurity atoms or the crystal structure. Indeed, austenitic steels of significantly different compositions and swelling incubation periods exhibit similar steady-state swelling rates of $\sim 1 \%$ per NRT displacement per atom (dpa) [27]. And, although generally the bcc materials show remarkable resistance to swelling [27,28], the alloy V$5 \%$ Fe showed the highest swelling rate of 3\% per dpa: 90\% at 30 dpa [29].

A key break-through in resolving some of the problems was due to formulation of the 'Production Bias Model' (PBM), first by Woo and Singh [30,31] and then, in its modern form, by Singh and coauthors [32-37]. The PBM enjoys advantages of the BEK theory and, in addition, succeeds in explaining several striking observations, such as high swelling rates at low dislocation density and near grain boundaries [32-34]; higher swelling rates in materials with smaller grain size [35]; the recoil energy effect, i.e. higher swelling rates under neutron compared to Frenkel-pair-producing electron irradiation [36,37]; and the absence of void lattices in $1 \mathrm{MeV}$ electron irradiated materials. The framework of the model also accounts for the minimum swelling rate in $\mathrm{Fe}-\mathrm{Cr}$ alloys at $\sim 10 \mathrm{at} \% \mathrm{Cr}$ [38] and the absence of copper precipitate growth in $\mathrm{Fe}-\mathrm{Cu}$ alloys at temperature less than $300^{\circ} \mathrm{C}$ [39]. The model owes its success to the recognition of two distinguishing features of defect production by high-energy recoils: first, the formation of thermally-stable clusters of self-interstitial atoms (SIAs) directly in displacement cascades, fact revealed both experimentally [40-44] and in molecular dynamics (MD) simulations [45-48]; and, second, the one-dimensional motion of the SIA clusters [46,49-52]. A fine review of the key experimental data and their analysis leading to formulation of the PBM can be found in [18].

Perhaps more important than explanation of any particular effect was a change of the entire concept of radiation damage theory by recognising that qualitatively different mechanisms operate in materials under, say, electron irradiation when the initial damage is in the form of the Frenkel pairs only, and neutron irradiation, when the continuous production of thermally-stable glissile SIA clusters in 
displacement cascades takes place. (The sessile SIA clusters are also produced but in our view are not as important in the analysis presented here.) Three comments can be made to facilitate appreciation of this concept. First, the formation of the SIA clusters under neutron irradiation is qualitatively different from that during $1 \mathrm{MeV}$ electron irradiation. Indeed, the nucleation of the SIA clusters under electron irradiation is limited to relatively small irradiation doses, when the total sink strength of the lattice defects including immobilised SIA clusters is low enough for the rate of the association reactions between two SIAs to be sufficiently high. In contrast, the cascade production of the SIA clusters operates at all times. Second, like the PBM, the BEK model also accounts for clustering of defects produced in cascades, but only vacancies in the form of small vacancy loops. Consequently, it contributes to the description of the microstructure evolution, but only at low temperatures, when the vacancy loops are thermally stable. In contrast, the SIA clusters are thermally stable at all temperatures of practical importance. Thus, since BEK model does not consider production of SIA clusters, it cannot describe damage accumulation properly even at low temperatures because it cannot include the consequence of 1-D diffusion of SIA clusters. Third, unlike the BEK theory, in the PBM it is crucial that some fraction of the SIA clusters is mobile and escapes to various sinks. Otherwise, accumulation of an extremely high density of these clusters would occur, known as 'Singh-Foreman catastrophe' [32], leading to complete termination of any further evolution of the microstructure. In contrast, in the BEK theory, the accumulation of a high density of vacancy clusters is only possible at sufficiently low temperature, due to their low thermal stability.

The successful applications of the PBM so far have been limited to low irradiation doses ( $<1 \mathrm{dpa})$ and pure metals (e.g. copper). Furthermore, the PBM in its present form predicts that the void swelling should come to saturate at some high dose level. This prediction originates from the mixture of onedimensional and three-dimensional reaction kinetics under cascade damage conditions, hence from the assumption lying at the heart of the model, i.e. the production of one-dimensionally (1-D) migrating SIA clusters in cascades. More specifically, it stems from the fact that the interaction cross-section with a void is proportional to the void radius, $r$, for 3-D migrating vacancies and to $r^{2}$ for 1-D diffusing SIA clusters. As a result, above some critical radius, the latter becomes higher than the former and the net vacancy flux to such voids negative.

In contrast, experiments demonstrate unlimited void growth at high doses for a majority of materials and irradiation conditions (see e.g. [27-29]). Thus, even the PBM fails to account for the most important observation under neutron or heavy ion irradiation. Some specific mechanisms could operate in different materials, but the observed unlimited damage accumulation is a common feature in all materials. So, while the lattice type and the presence of solute and impurities atoms distinguish one material from another, there must be general reasons for the observed unlimited defect accumulation, common for all materials. Revealing these reasons seems to be a central problem of the radiation damage physics and is the main objective of the present work.

To solve the problem we propose to reconsider the assumptions of the PBM which are proved to be reasonable at low doses but may be completely unrealistic at higher doses and for alloys. It is assumed, for example, that the spatial distribution of voids and properties of void surfaces, the 1-D transport behaviour of SIA clusters and the evolution of dislocation microstructure remain unaltered during continuous irradiation to high doses. However, even in the case of pure metals, complications may arise at higher doses because of continuous production of transmutational impurities during neutron irradiation. The presence of impurity atoms and alloying elements is likely to modify the behaviour of voids as well as dislocations as sinks because of segregation of impurity atoms and affect the diffusivities of both 3-D migrating point defects and 1-D migrating SIA clusters. Other complications may be due to development of spatial correlations between different type defects in the form of void super-lattices, dislocation walls, decoration of dislocations with loops, association of voids with second-phase precipitates and dislocations (see Section 4.1 for further discussion). All these may lead to incorrect description of the reaction kinetics and thereby the nature and magnitude of damage accumulation. It is 
therefore vitally important to include realistic treatments of the issues mentioned above into the PBM and make it suitable for predicting the microstructure evolution and mechanical properties of technological materials under reactor relevant operational conditions.

The paper is organised as follows. In Section 2, it is shown that voids can grow indefinitely if the production bias, i.e. the fraction of SIAs produced in cascades in the form of 1-D mobile clusters is smaller than a certain value of the order of the dislocation bias, but argued that this condition is unlikely to be satisfied for neutron irradiation. In Section 3, the conditions for the onset of void lattice formation are analysed and it is argued that spatial correlations of voids with each other and with other defects must be a common feature in all materials and be always present under cascade irradiation. The development of these correlations minimises the void-SIA cluster interaction intensity, and leads to screening of voids from the SIA clusters. So, the void lattices represent just one type of spatial correlations possible and its very absence is an indication that correlations of other types prevail. In Section 4, the theory is generalised for inclusion of the correlation-screening effects and is shown to explain the unlimited void growth and provide a simple interpretation of the universal swelling rates observed in stainless steels. In Section 5, it is shown that development of solute and impurity atmospheres around voids, which repel the SIA clusters, may assist or even solely explain the unlimited void growth. A summary and outlook are given in Section 6. 


\section{GROWTH AND SATURATION OF VOIDS DISTRIBUTED RANDOMLY}

In this section, an equation for the void saturation radius in conditions when both the production and dislocation bias operate is derived. It is assumed for simplicity that the primary damage produced in cascades of atomic displacements consists of 3-D mobile single vacancies and SIAs and 1-D mobile SIA clusters only. The fraction of the SIAs in the latter, $\varepsilon_{\mathrm{i}}^{\mathrm{g}}$, represents the production bias factor in such a model. The void growth is considered after the end of the void nucleation stage when the mobile defects interact only with existing voids of a number density $N$ and edge dislocations of a density $\rho$. All defects are assumed to be distributed randomly over the volume. Then, according to the PBM (see, e.g. in [35]), the swelling rate, $d S / d \phi\left(S=4 \pi r^{3} N / 3\right)$, is equal to the difference in the arrival rates of vacancies, single SIAs and SIAs in clusters to voids:

$$
\frac{d S}{d \phi}=\varepsilon_{\mathrm{i}}^{\mathrm{g}}\left(\frac{4 \pi r N}{4 \pi r N+Z_{\mathrm{v}} \rho}-\frac{\pi r^{2} N}{\pi r^{2} N+\pi \rho r_{\mathrm{d}} / 2}\right)+\left(1-\varepsilon_{\mathrm{i}}^{\mathrm{g}}\right)\left(\frac{4 \pi r N}{4 \pi r N+Z_{\mathrm{v}} \rho}-\frac{4 \pi r N}{4 \pi r N+Z_{\mathrm{i}} \rho}\right),
$$

where $\phi=\varepsilon_{\text {surv }} \phi^{\mathrm{NRT}}$ is the irradiation dose in dpa, which takes into account the fraction of defects that survive intra-cascade recombination ( $\varepsilon_{\text {surv }}=1-\varepsilon_{\mathrm{r}}, \varepsilon_{\mathrm{r}}$ being the fraction of point defects recombining during the cooling stage of cascades [34]), $Z_{\mathrm{v}}$ and $Z_{\mathrm{i}}$ are the capture efficiencies of edge dislocations for vacancies and single SIAs, respectively, and $r_{\mathrm{d}}$ is the dislocation capture radius for the SIA clusters. In the right-hand side of equation (1), the swelling rate is divided into two terms proportional to the fractions of 1-D and 3-D migrating SIAs, respectively. If all the SIAs were produced in the clustered form and migrated 1-D, then only the first term would exist and the swelling would be terminated when the void mean radius reached the value $r_{\mathrm{m} 0}=2 \pi r_{\mathrm{d}} / Z_{\mathrm{v}}$ [33]. If, on the other hand, no 1-D migrating clusters are formed, as under electron irradiation, then only the second term would be present and unlimited void growth would be observed, with a rate proportional to the dislocation bias factor $B=Z_{\mathrm{i}} / Z_{\mathrm{v}}-1$, which originates from a stronger interaction of single SIAs than vacancies with edge dislocations [11].

In general, the two terms interplay and the swelling rate becomes negative for voids with radius bigger than the following critical value

$$
r_{\mathrm{m}}=r_{\mathrm{m} 0} F\left(B / \varepsilon_{\mathrm{i}}^{\mathrm{g}}\right)
$$

where

$$
F(x)=\frac{2(1+x) /(1+B)}{1-\alpha \pm \sqrt{(1-\alpha)^{2}+4 \alpha\left[1-(\gamma x)^{2}\right]}},
$$

$\alpha=4 \pi r_{\mathrm{m} 0} N / Z_{\mathrm{i}} \rho$ and $\gamma^{2}=\left(1-\varepsilon_{\mathrm{i}}^{\mathrm{g}}\right) /(1+B)$. The parameter $\alpha$ is the ratio of sink strengths of voids and dislocations for 3-D migrating defects in the saturation regime. Note that $\alpha=\pi r_{\mathrm{m} 0}^{2} N /\left(\pi r_{\mathrm{d}} \rho / 2\right)(1+B)$, i.e. with a good accuracy it is equal to the same ratio for 1-D migrating defects. Equation (2) represents general solution corresponding to zero swelling rate. There can be no, one or two solutions for the saturation radius, depending on the values of the parameters $\alpha$ and $\varepsilon_{\mathrm{i}}^{\mathrm{g}}$. 
So, when $\alpha \geq 1$ (voids are dominant sinks for both 1-D and 3-D migrating defects), there is no solution, corresponding to unlimited void growth, if $\varepsilon_{\mathrm{i}}^{\mathrm{g}} \leq \gamma B$, or one solution, if $\varepsilon_{\mathrm{i}}^{\mathrm{g}}>\gamma B$. In the latter case, the critical radius is given by the '+' sign in equation (3) and increases with increasing $B / \varepsilon_{\mathrm{i}}^{\mathrm{g}}$, approaching infinity when $\varepsilon_{\mathrm{i}}^{\mathrm{g}} \rightarrow \gamma B$. Figure 1 shows this dependence for $\alpha=10$ and $B=0.04$. In this figure, regions of void dissolution and of limited and unlimited void growth are indicated.

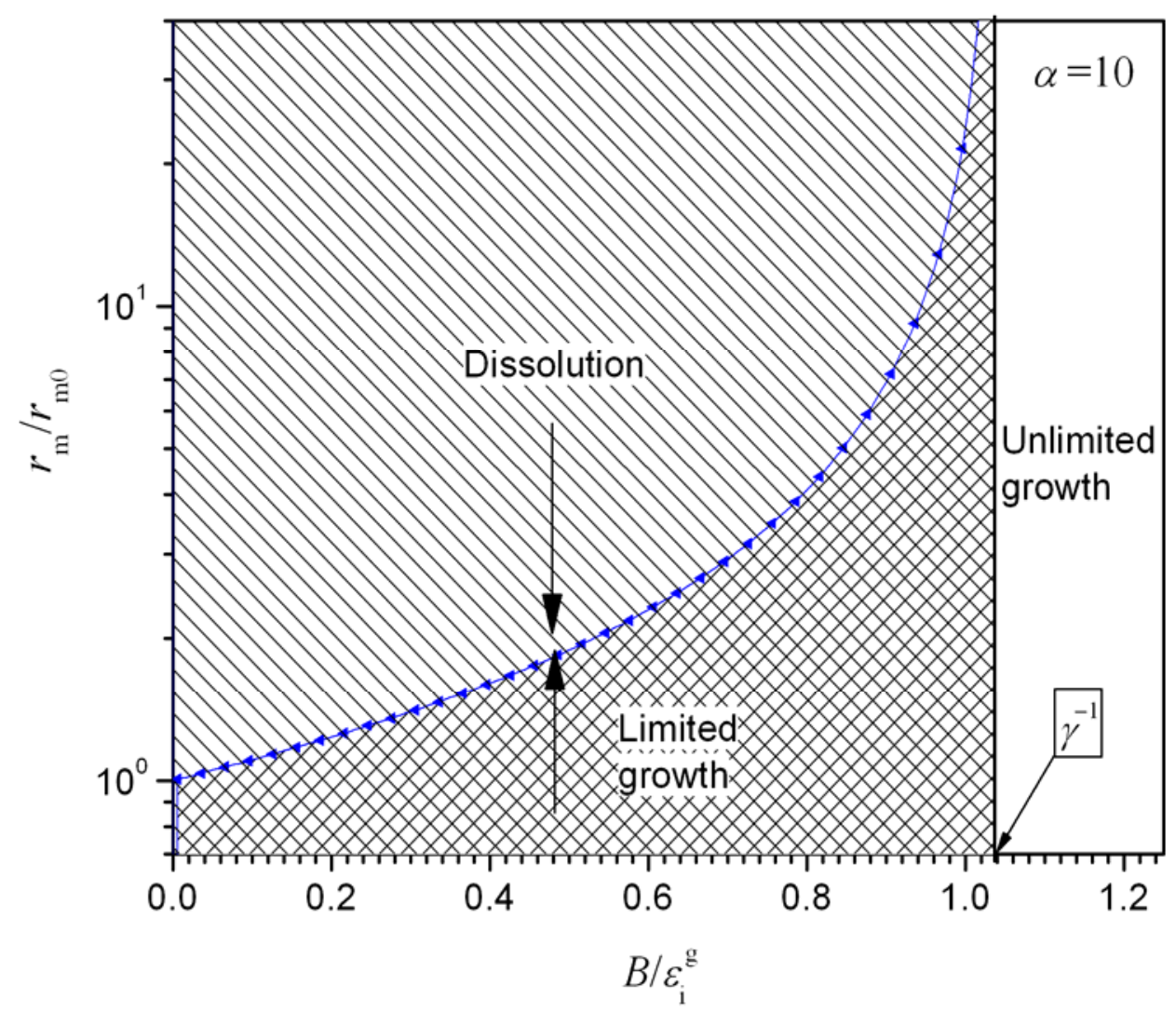

Figure 1. The dependence of the saturated void radius on the ratio of the dislocation bias and the fraction of SIAs produced in the form of 1-D migrating SIA clusters, calculated for $\alpha=10$ and $B=0.04$.

When $\alpha<1$ (edge dislocations are dominant sinks), the dependence of the steady-state void radius on the ratio of the dislocation and production bias factors is shown in figure 2. (See also figure 3 for more calculation results.) As seen from figure 2, there is no swelling saturation if the fraction of 1-D diffusing SIAs is smaller than some finite value: $\varepsilon_{\mathrm{i}}^{\mathrm{g}}<2 B \gamma /\left(\alpha^{1 / 2}+\alpha^{-1 / 2}\right)$. For $\varepsilon_{\mathrm{i}}^{\mathrm{g}}>B \gamma$, there is one stable solution, while for the intermediate values of $\varepsilon_{\mathrm{i}}^{\mathrm{g}}$, there are two, one stable and one unstable, solutions. The stable solutions correspond to '+' and the unstable to '-' sign in equation (3). The unstable solution defines a dividing line between regions of dissolution and unlimited void growth. 


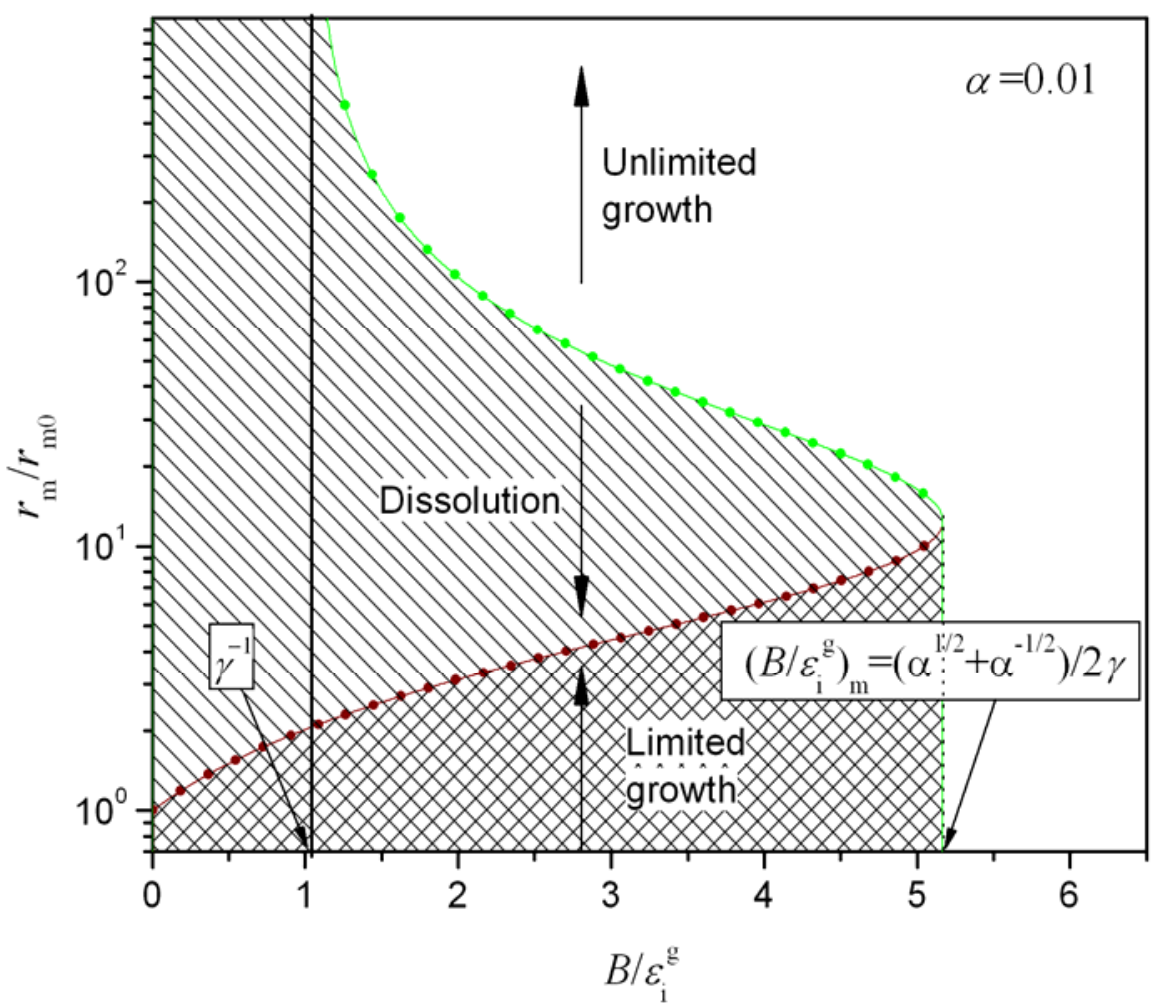

Figure 2. Same as in figure 1 but for $\alpha=0.1$.

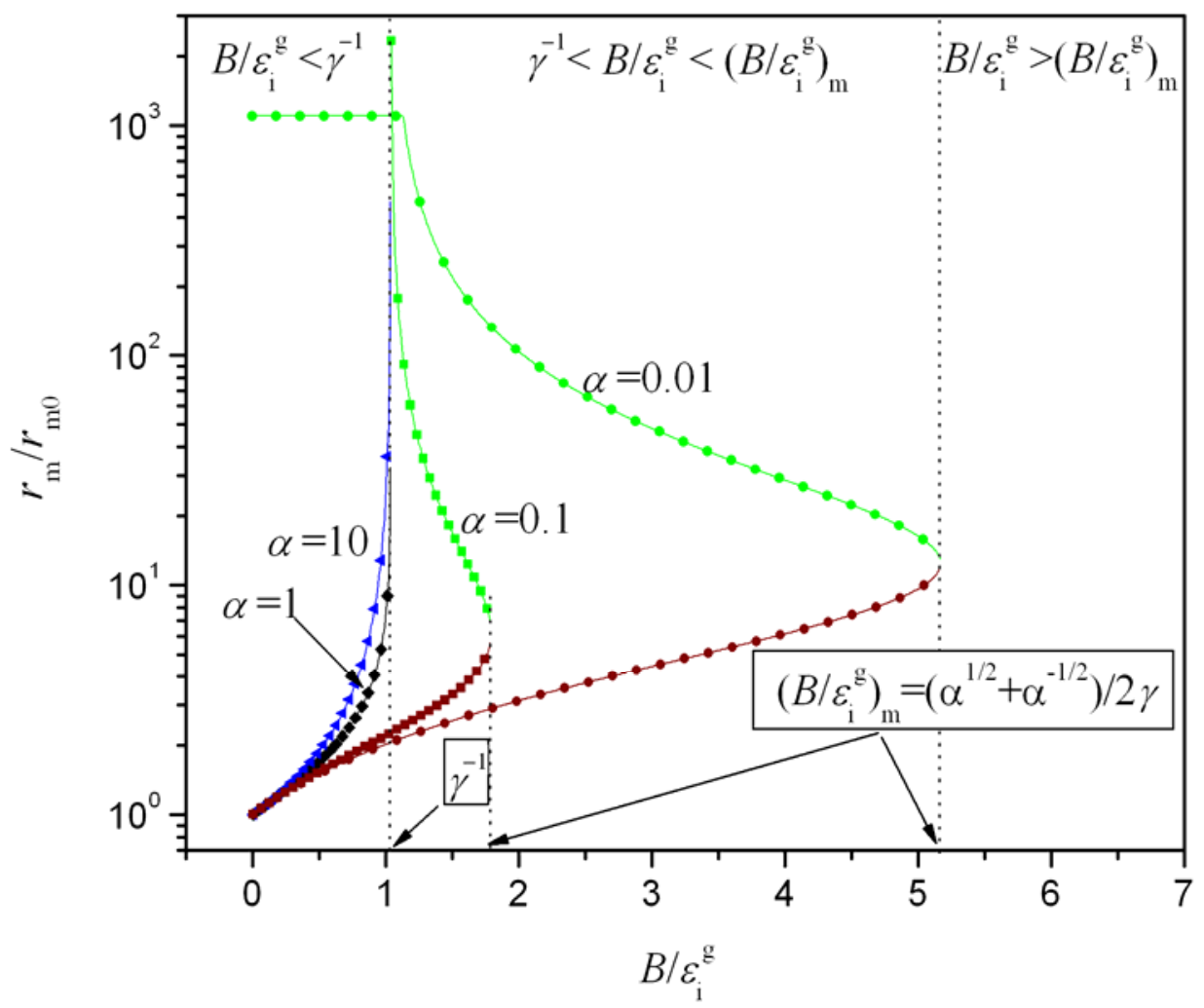

Figure 3. Same as in figure 1 but for different $\alpha$. 
Summarising the results presented above, we conclude that an unlimited growth of randomly distributed voids is possible if the fraction of 1-D diffusing defects is smaller than some finite value of the order of the dislocation bias factor, $B$ :

$$
\varepsilon_{\mathrm{i}}^{\mathrm{g}}< \begin{cases}B \gamma, & \alpha \geq 1, \\ B \gamma \frac{2}{\alpha^{1 / 2}+\alpha^{-1 / 2}}, & \alpha<1 .\end{cases}
$$

It is known that the value of $\varepsilon_{\mathrm{i}}^{\mathrm{g}}$ depends on the recoil energy and, according to MD simulations, is $\sim 0.6$ in iron and copper for the primary knock-on atom energy $\sim 10 \mathrm{keV}$ [47]. These simulations are of nanosecond scale and the defects produced are still confined within a small region of $\sim 10 \mathrm{~nm}$ size, so that the annealing that follows should result in additional clustering and recombination reactions that might change the fraction $\varepsilon_{\mathrm{i}}^{\mathrm{g}}$. There seem to be a scatter of opinions on the value of the dislocation bias, $B$. Estimates produced using elasticity theory considered the edge dislocation-point defect interactions introduced by Cottrell (the first order size effect) [53] and Eshelby (the stress-induced interaction) [54] and resulted in $B \approx 0.2 \div 0.5$ [55,56]. If these values were correct, the dislocation bias would be able to compete with the production bias and explain the unlimited void growth. However, fitting the SRT to available experimental data on swelling in electron-irradiated materials requires $B$ to be an order of magnitude smaller, $\sim 0.02$ [37,57]. It is generally believed that the SRT is quite capable of describing void swelling in this case, so that the small values of $B$ should deserve more trust. Hence, it is unlikely that condition (4) is satisfied under cascade-produced irradiation. To elucidate the reasons behind the discrepancy between different approaches for estimating the dislocation bias, this area should be revisited. 


\section{ONSET OF SPATIAL CORRELATIONS DUE TO 1-D MIGRATING SIA CLUSTERS}

Another possibility for the unlimited void growth can be provided by development of spatial correlations either between voids or between voids and other lattice defects, such as dislocations and precipitates, that shadow voids from the SIA clusters. Below, in the next two sections, we argue that this must be the case. The analysis is started with specific but already well-known spatial correlations between voids in the form of a void lattice. This phenomenon is observed in a number of bcc metals (Mo, $\mathrm{W}, \mathrm{Nb}, \mathrm{Ta}$ ), the fcc metals $\mathrm{Ni}$ and $\mathrm{Al}$, the hcp Mg, and some alloys, e.g. stainless steel, under neutron and heavy-ion irradiations (see e.g. in a review [58]). In an accompanying paper [59] the conditions for the onset of void ordering in metals under cascade irradiation are analysed by using a mathematical approach of Helbing and Vicsek [60] developed for the description of lane formation in pedestrian crowds. To make the present paper self-consistent, below the method is briefly outlined and the main conclusions are described.

The physical model is the same as described in the beginning of the previous section. With the total void density assumed to be constant, the local void density changes due to voids leaving and entering the local region and hence obeys a continuity equation

$$
\frac{\partial N}{\partial \phi}=-\operatorname{div}(N \mathbf{V}-D \nabla N)
$$

where $\mathbf{V}=\left\langle\sum_{j} v_{j} \Delta_{j}\right\rangle$ is the mean velocity and $D=\frac{1}{6}\left\langle\sum_{j} v_{j} \Delta_{j}^{2}\right\rangle$ is the diffusion coefficient of voids, defined locally. Here, $j=\mathrm{v}, \mathrm{i}, \mathrm{cl}$ for the components corresponding to collisions of voids with vacancies, single SIAs and SIAs in clusters, respectively, $v_{j}=p_{j} / \Omega N$ are the collision frequencies, $\Omega$ being the atomic volume, $\Delta_{j}$ are the vectors of void displacements after collisions and the averaging is performed over voids in the local area. In [59], the expressions for the void velocity and diffusion coefficient through the microstructure parameters and the basic conditions for destabilisation of homogeneous solution, $N^{\text {hom }}$, of equation (5) are derived. For this the linear stability analysis is applied. Using the ansatz $N=N^{\text {hom }}+\tilde{N} \exp (i \mathbf{q R}+a \phi)$, where $\mathbf{R}$ is the radius vector, $\mathbf{q}$ is a wave vector and $a$ is the amplification factor, the most favourable conditions for destabilisation are found to be for a wave vector satisfying the following conditions

$$
\begin{aligned}
& r<<q^{-1}<<L_{3 \mathrm{D}}, \\
& \mathbf{q} \|\left[\mathbf{b}_{k}, \mathbf{b}_{k^{\prime}}\right],
\end{aligned}
$$

where $\mathbf{b}_{k}$ and $\mathbf{b}_{k^{\prime}}\left(k \neq k^{\prime}\right)$ are any two different Burgers vectors of the SIA clusters and $L_{3 \mathrm{D}} \approx\left(4 \pi r N+Z_{\mathrm{v}} \rho\right)^{-1 / 2}$ is the mean free path of 3-D migrating point defects. These conditions define the void movement leading to formation of close-packed planes $\left[\mathbf{b}_{k}, \mathbf{b}_{k^{\prime}}\right]$ of voids, which is consistent with the observed isomorphism of the void and host lattices. Note that the mean-free path of 1-D migrating clusters, $L_{1 \mathrm{D}}$ (see, e.g. in [61]): 


$$
L_{1 \mathrm{D}}=\left(\pi r^{2} N+\pi \rho r_{\mathrm{d}} / 2\right)^{-1},
$$

does not enter explicitly the conditions (6) and (7). The destabilisation of the homogeneous solution due to condition (7) is only possible, however, if the concentration fields of the SIA clusters around distant voids overlap, which require big $L_{1 \mathrm{D}}>L_{3 \mathrm{D}}$.

The conditions for the onset of void ordering are then derived, which can be written using parameters defined in the present paper and assuming $Z_{\mathrm{i}}=Z_{\mathrm{v}}=1$ as

$$
\frac{4 \pi r^{3}}{3 \Omega}>\frac{n m}{3}\left[1+\frac{1}{\alpha\left(r / r_{\mathrm{m}}\right)^{2}}\right]\left[1+\frac{2-\varepsilon_{\mathrm{i}}^{\mathrm{g}}}{\varepsilon_{\mathrm{i}}^{\mathrm{g}} m} \frac{1+\alpha\left(r / r_{\mathrm{m}}\right)^{2}}{1+\alpha\left(r / r_{\mathrm{m}}\right)} \frac{r}{r_{\mathrm{m}}}\right],
$$

where $n=4$ for the bcc and $n=6$ for the fcc crystal lattice is the number of different Burgers vectors and $m$ is the mean number of SIAs in a cluster. Equation (9) predicts that, for low enough dislocation density, spatial correlations between voids should always be present, except for very early stages of irradiation, when voids are too small, of the order of ten vacancies (see figure 1 in [59]).

The spatial correlations between voids are possible, of course, if correlations with other defects of a higher density do not prevail. We believe that we know why voids sometimes form super-lattices, although not all aspects of the process are yet well understood, e.g. the reason for low swelling rates or even saturation of swelling observed in void lattices. So, the real question is why it does not happen every time? We propose that spatial correlations always develop under cascade damage conditions. The void lattices represent only one type, which is realised when the void number density is high enough. The very absence of a void lattice must be an indication of the existence of correlations of voids with other defects. At lower void density, spatial correlations with other defects, e.g. precipitates and dislocations, are formed. Which correlations prevail depends on the densities of different defects as well as some other properties as also discussed in the next section. In the analysis presented above it is assumed that voids are correlated with each other rather than with other elements of microstructure. For a system containing voids and edge dislocations, this assumption is satisfied if the probability that an SIA cluster is generated between two voids is higher than that between a void and a dislocation, that is, if [59]

$$
r^{2} N>\rho r_{\mathrm{d}} .
$$

It is proposed in [59] that this is the reason why the void lattice formation is observed at high void and low dislocation density, rather than due to the limitations imposed by equation (9). This also implies that other microstructure features, e.g. dislocations and precipitates, must be involved in shadowing voids from the 1-D mobile SIA clusters and establishing spatial correlations. So, for example, considering void movement in the presence of a gradient of dislocation density and representing the spatial dependence of the dislocation density in the form $\rho=\rho^{\text {hom }}+\tilde{\rho} \exp \left(i \mathbf{q}_{d} \mathbf{R}\right)$, an equation for the amplification factor can be obtained as

$$
a=\frac{\tilde{\rho}}{\tilde{N}} q_{\mathrm{d}}^{2} A_{\mathrm{d}}^{2} \exp \left[i\left(\mathbf{q}_{\mathrm{d}}-\mathbf{q}\right) \mathbf{R}\right]-q^{2} D,
$$


where $A_{\mathrm{d}}$ is a function of microstructure, such as void and dislocation densities, and other parameters. The first term in the right-hand side of equation (11) is positive, thus providing most favourable conditions for destabilisation of the homogeneous solution, $\rho^{\text {hom }}$, if $\mathbf{q}=\mathbf{q}_{\mathrm{d}}$, i.e. when void positions correlate with that of dislocations.

This section can be summarised as follows: spatial correlations between voids and either voids or other microstructure elements must almost always be present under cascade-damage conditions. The prevalence of specific spatial correlations, e.g. between voids or between voids and other defects, such as precipitates and dislocations, should be governed by corresponding defect densities. In the next section we argue that the development of such correlations must be one of the key mechanisms responsible for the unlimited damage accumulation observed in reactor materials. 


\section{EFFECT OF CORRELATIONS ON SWELLING RATE}

\subsection{Background}

It follows from the preceding section that a predictive theory of damage accumulation under cascade-damage conditions must account for the effect of spatial correlations on screening of voids from the SIA clusters. It is worth mentioning that already in 1970 serious doubts were expressed by Farrell and Houston [62] on the validity of the void nucleation theories based on homogeneous nucleation. Later, Singh and Leffers [23] concluded that a new concept is needed for treating the problem of defect accumulation under cascade damage conditions, and then Krishan et al. [63] made first attempt to extend the theory beyond the conventional mean-field approach.

Experimental evidence on association of large voids with various precipitates ( $G$, $\eta$, Laves, etc.) [12,64-68] and growth of voids in the compression side of edge dislocations (see e.g. [62,69-70]) has been known for a long time. More recent evidence is due to Kozlov, Portnykh et al. [71,72] and Xu et al. [73]. In the latter work, association between copper precipitates and vacancy clusters was revealed, which we propose can be due to screening of voids from the SIA clusters by the precipitates. Kozlov, Portnykh et al. [71,72] observed spatial correlations in $20 \%$ cold-worked $16 \mathrm{Cr}-15 \mathrm{Ni}-2 \mathrm{Mo}-2 \mathrm{Mn}$ austenitic steel irradiated up to 100 dpa irradiation dose in a BN-600 fast reactor in the temperature range from 410 to $600^{\circ} \mathrm{C}$. Transmission electron microscopy revealed voids of three main types: a-type associated with dislocations, b-type associated with $G$-phase precipitates and c-type distributed homogeneously in the matrix. The c-type voids were the smallest in size and made practically no contribution to swelling, while the a-type voids were the largest. Note the high irradiation doses in these experiments, which might be the reason why the association was detectable. As argued in the previous section, such spatial correlations must be a common feature in all materials under cascade-irradiation conditions and present even at low irradiation doses. The difficulty of observing them in most cases is due to much larger scale of 1-D than 3-D correlations. Indeed, common perception restricts spatial correlations to those developing on a scale of the order of the average distance between defects, $L_{3 \mathrm{D}}$, which is typically $\sim 100 \mathrm{~nm}$. So, in a void lattice, the presence of correlations is obvious. In contrast, the mean-free path of 1-D migrating SIA clusters, $L_{1 \mathrm{D}}$, equation (8), is typically $\sim \mu \mathrm{m}$ or even longer, especially at low irradiation dose, when the size and the number density of defects are still small. As irradiation proceeds, $L_{1 \mathrm{D}}$ decreases due to increase of the defect density and/or size, getting closer to $L_{3 \mathrm{D}}$. This is one of the reasons why spatial correlations at high irradiation dose should be easier to detect.

The development of spatial correlations of voids with precipitates, which repel the SIA clusters, seems quite natural: the nucleation and survival of a void in the vicinity of and in one of the close-packed directions from a precipitate should be much easier than in the matrix away from any defects, because of partial or complete shadowing of the void by the precipitate from the SIA clusters. A small precipitate with no long-range strain field would block only one crystallographic direction for the arrival of SIA clusters to the void. This may significantly enhance the formation rate of void nuclei, since it is highly sensitive to the difference between vacancy and interstitial fluxes. However, it will be shown below that this would increase the maximum void size only slightly. Simple geometrical screening would allow a large precipitate to block, on average, up to half of all the directions. If, in addition, the precipitate repels the SIA clusters due to long-range strain field, the void would not interact with the SIA clusters at all until its size exceeds the range of strain field.

The growth of voids in the compression sides of edge dislocations is another possibility of void screening, although it appears initially more difficult to justify due to the possibility of dislocation climb, which would break the void-dislocation correlation. Clearly, this situation is not simple and requires detailed investigation, which takes into account the influence of elastic interaction between voids and 
dislocations on dislocation climb. In any case, repulsive barriers for the SIA clusters due to precipitates and compression regions of edge dislocations have to be introduced into the theory.

To reveal the effect of repulsive barriers and screening of voids on the void growth, void swelling is considered below in three situations: (a) in the absence of repulsive barriers, (b) with repulsive barriers but in the absence of spatial correlations and (c) with spatial correlations present. Random distribution of dislocations and repulsive barriers is assumed in all cases and dislocation bias is neglected for simplicity.

\subsection{Void swelling in the absence of repulsive barriers}

For the situation (a), i.e. in the absence of repulsive barriers and randomly distributed voids, the swelling rate is described by equation (1), which we simplify by neglecting the dislocation bias, that is by taking $Z_{\mathrm{i}}=Z_{\mathrm{v}}$,

$$
\frac{d S}{d \phi}=\varepsilon_{\mathrm{i}}^{\mathrm{g}}\left[\frac{\alpha\left(r / r_{\mathrm{m}}\right)}{1+\alpha\left(r / r_{\mathrm{m}}\right)}-\frac{\alpha\left(r / r_{\mathrm{m}}\right)^{2}}{1+\alpha\left(r / r_{\mathrm{m}}\right)^{2}}\right]=\varepsilon_{\mathrm{i}}^{\mathrm{g}}\left(p_{3 \mathrm{~V}}-p_{1 \mathrm{~V}}\right) .
$$

This equation defines $p_{3 \mathrm{~V}}$ and $p_{1 \mathrm{~V}}$, the probabilities that a 3-D migrating vacancy and a 1-D migrating SIA produced in a cascade are absorbed by a void.

\subsection{Void swelling in the presence of repulsive barriers not correlated with voids}

For situation (b), let us define the probability that a cluster is absorbed by a void, that is $p_{1 \mathrm{~V}}$ in equation (2). Consider the probabilities that an SIA cluster is produced between two sinks/barriers of particular types. These are proportional to the products of the cross-sections of those sinks/barriers: $\sigma_{\text {bar }}$

for repulsive barriers $\left(=\sigma_{\mathrm{ppt}} \equiv \pi r_{\mathrm{ppt}}^{2} N_{\mathrm{ppt}}\right.$ for precipitates or $\propto \rho$ for compression regions of edge dislocations), $\sigma_{\mathrm{V}}=\pi r^{2} N$ for voids and $\sigma_{\mathrm{d}}=\pi \rho r_{\mathrm{d}} / 2$ for attractive part of dislocations. These proportionalities are collected in table 1, together with the total sum, which is the normalising constant for the corresponding probabilities, and the partial sum for voids. The total is obtained as a sum of all values from (i) to (vi), while the partial value for the SIA clusters interacting with voids only is the sum of (ii), (iii) and one half of (v). The half occurs because half of the SIA clusters produced between a void and a dislocation must be captured by the dislocation. The ratio of the partial to the total equals the probability that a cluster is absorbed by a void, $p_{1 \mathrm{~V}}$. In this way, the swelling rate is obtainable from equation (12) by replacing

$$
\begin{aligned}
& p_{1 \mathrm{~V}} \rightarrow \frac{\sigma_{\mathrm{V}}\left(\sigma_{\mathrm{d}}+2 \sigma_{\mathrm{bar}}+\sigma_{\mathrm{V}}\right)}{\left(\sigma_{\mathrm{d}}+\sigma_{\mathrm{bar}}+\sigma_{\mathrm{V}}\right)^{2}}, \\
& p_{3 \mathrm{~V}} \rightarrow p_{3 \mathrm{~V}}\left[1-\frac{\sigma_{\mathrm{ppt}}^{2}}{\left(\sigma_{\mathrm{d}}+\sigma_{\mathrm{ppt}}+\sigma_{\mathrm{V}}\right)^{2}}\right] .
\end{aligned}
$$


Table 1. Determination of probabilities of different reactions for 1-D mobile SIA clusters in a system containing voids, dislocations and repulsive barriers (subscript 'bar').

\begin{tabular}{lll}
\hline i & Two barriers & $\sigma_{\text {bar }}^{2}$ \\
ii & A void and a barrier & $2 \sigma_{\mathrm{v}} \sigma_{\text {bar }}$ \\
iii & Two voids & $\sigma_{\mathrm{V}}^{2}$ \\
iv & Two dislocations & $\sigma_{\mathrm{d}}^{2}$ \\
v & A dislocation and a void & $2 \sigma_{\mathrm{d}} \sigma_{\mathrm{v}}$ \\
vi & A dislocation and a barrier & $2 \sigma_{\mathrm{d}} \sigma_{\mathrm{bar}}$ \\
& Total sum & $\left(\sigma_{\mathrm{d}}+\sigma_{\mathrm{bar}}+\sigma_{\mathrm{v}}\right)^{2}$ \\
& Partial sum for voids & $\sigma_{\mathrm{v}}\left(\sigma_{\mathrm{d}}+2 \sigma_{\mathrm{bar}}+\sigma_{\mathrm{v}}\right)$ \\
\hline
\end{tabular}

Equation (13) has already been explained above, while equation (14) accounts for the SIA clusters captured between two repulsive barriers. In the framework of the model considered, where high vacancy super-saturation exists, such clusters must eventually recombine with freely migrating vacancies. The factor in the brackets of equation (14) excludes the corresponding fraction of vacancies from reactions with voids. The resulting equation for the swelling rate is

$$
\frac{d S}{d \phi}=\varepsilon_{\mathrm{i}}^{\mathrm{g}} p_{3 \mathrm{~V}} \frac{1+\left(\sigma_{\mathrm{V}}+2 \sigma_{\mathrm{bar}}\right) / \sigma_{\mathrm{d}}}{\left[1+\left(\sigma_{\mathrm{V}}+\sigma_{\mathrm{bar}}\right) / \sigma_{\mathrm{d}}\right]^{2}}\left[1-\left(p_{3 \mathrm{~V}}^{-1}-1\right) \frac{\sigma_{\mathrm{V}}}{\sigma_{\mathrm{d}}}\right] \text {. }
$$

Taking into account that $\sigma_{\mathrm{V}} / \sigma_{\mathrm{d}}=\alpha\left(r / r_{\mathrm{m}}\right)^{2}$ and $p_{3 \mathrm{~V}}=\alpha\left(r / r_{\mathrm{m}}\right) /\left[1+\alpha\left(r / r_{\mathrm{m}}\right)\right]$ (see equation (12)), one finally obtains

$$
\frac{d S}{d \phi}=\frac{\varepsilon_{\mathrm{i}}^{\mathrm{g}} p_{3 \mathrm{~V}}\left(1-r / r_{\mathrm{m}}\right)}{1+\alpha\left(r / r_{\mathrm{m}}\right)^{2}+\sigma_{\mathrm{bar}} / \sigma_{\mathrm{d}}}\left[1+\frac{\sigma_{\mathrm{bar}} / \sigma_{\mathrm{d}}}{1+\alpha\left(r / r_{\mathrm{m}}\right)^{2}+\sigma_{\mathrm{bar}} / \sigma_{\mathrm{d}}}\right] .
$$

As can be seen from this equation, the steady-state saturation radius of voids is the same as without repulsive barriers. The effect of randomly distributed barriers is a decrease in the swelling rate by enhancing recombination of the SIA clusters with freely migrating vacancies. The swelling rate tends to zero in the limit $\sigma_{\text {bar }} \rightarrow \infty, e . g$. for large number density of precipitates. Thus, the presence of randomly distributed repulsive barriers in the matrix decreases the swelling rate but does not affect the void saturation radius.

\subsection{Void swelling in the presence of correlations}

Situation (c), when spatial correlations exist, is similar to situation (b). The only difference is in the cross-sections of the repulsive barriers and voids. Equation (15) is still valid provided that correct cross-sections are used. Let us introduce parameters $\eta_{\text {bar }}$ and $\eta_{\mathrm{v}}$, which we call 'correlation-screening factors', such that the products $\eta_{\mathrm{bar}} \sigma_{\mathrm{bar}}$ and $\eta_{\mathrm{v}} \sigma_{\mathrm{v}}$ give the correct cross-sections. The case $\eta_{\mathrm{v}}=0$ corresponds to complete screening of voids from the SIA clusters and $\eta_{\mathrm{V}}=1$ to the case of randomly distributed voids, and similarly for repulsive barriers. By substituting these into equation (15), one obtains a generalised version of equation (16) 


$$
\frac{d S}{d \phi}=\varepsilon_{\mathrm{i}}^{\mathrm{g}} p_{3 \mathrm{~V}} \frac{1+\eta_{\mathrm{V}} \alpha\left(r / r_{\mathrm{m}}\right)^{2}+2 \eta_{\mathrm{bar}} \sigma_{\mathrm{bar}} / \sigma_{\mathrm{d}}}{\left[1+\eta_{\mathrm{v}} \alpha\left(r / r_{\mathrm{m}}\right)^{2}+\eta_{\mathrm{bar}} \sigma_{\mathrm{bar}} / \sigma_{\mathrm{d}}\right]^{2}}\left(1-\eta_{\mathrm{v}} \frac{r}{r_{\mathrm{m}}}\right)
$$

As can be seen from this equation, the screening of voids results in an increase of swelling rate and the saturation radius, $\tilde{r}_{\mathrm{m}}$, which is defined by rescaling:

$$
\tilde{r}_{\mathrm{m}}=r_{\mathrm{m}} / \eta_{\mathrm{v}}
$$

It follows from this equation that unlimited void growth is possible if voids are completely screened from the SIA clusters, i.e. when $\eta_{\mathrm{V}}=0$. This can be realised, e.g. when voids grow near big precipitates with long-range strain field, repulsive for the SIA clusters, or in the compression sides of edge dislocations. The voids can grow then until their size exceeds the range of the repulsive field.

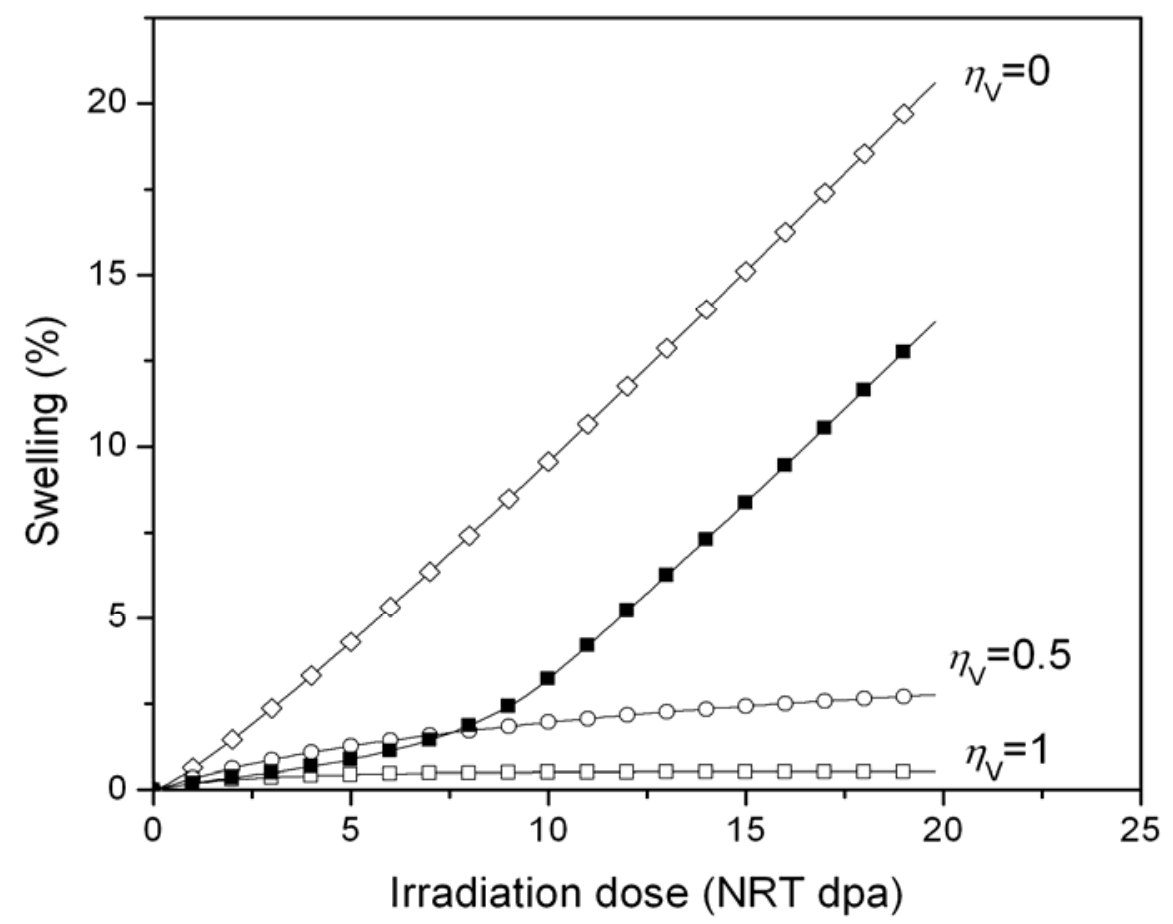

Figure 4. Dependence of swelling on irradiation dose calculated using equation (17) for $N=10^{22} \mathrm{~m}^{-3}$, $r_{\mathrm{m}}=5 \mathrm{~nm}, \varepsilon_{\mathrm{i}}^{\mathrm{g}}=0.2, \eta_{\mathrm{bar}} \sigma_{\text {bar }} / \sigma_{\mathrm{d}}=1, \varepsilon_{\text {surv }}=0.1$ and different values of the correlationscreening factor of voids, $\eta_{\mathrm{V}}$. The curve with full squares has been calculated for correlations developing with irradiation dose (see text).

Figure 4 presents the dependence of swelling on irradiation dose calculated using equation (17) for $N=10^{22} \mathrm{~m}^{-3}, r_{\mathrm{m}}=5 \mathrm{~nm}, \varepsilon_{\mathrm{i}}^{\mathrm{g}}=0.2, \rho \approx 6 \times 10^{14} \mathrm{~m}^{-2}$ (so that $\alpha=1$ ), $\eta_{\text {bar }} \sigma_{\text {bar }} / \sigma_{\mathrm{d}}=1$ and different values of the correlation-screening factor of voids, $\eta_{\mathrm{V}}$. The curve with full squares corresponds to the case, when spatial correlations develop with irradiation dose as: $\eta_{\mathrm{V}}=1-\phi^{\mathrm{NRT}} / 10$ when $\phi^{\mathrm{NRT}} \leq 10$ and $\eta_{\mathrm{V}}=1$ when $\phi^{\mathrm{NRT}}>10$. The survival fraction of defects in cascades was taken to be equal to $\varepsilon_{\text {surv }}=0.1$ (see definition after equation (1)) and the initial void radius was $0.25 \mathrm{~nm}$. As can be seen from the figure, with $\eta_{\mathrm{V}}=1$, it takes just several dpa for the swelling to saturate at a value corresponding 
to the void saturation radius $r_{\mathrm{m}}$. For $\eta_{\mathrm{V}}=0.5$, the saturation radius is twice as high and it takes longer for the saturation to occur. For $\eta_{\mathrm{V}}=0$, there is no saturation, i.e. voids grow indefinitely and the swelling rate is proportional to $\varepsilon_{\mathrm{i}}^{\mathrm{g}}$ (see further discussion below in relation to equation (23)).

\subsection{Calculation of correlation-screening factors}

Now let us calculate the parameters $\eta_{\text {bar }}$ and $\eta_{\mathrm{v}}$ for the case, when all voids are assumed to grow in the immediate vicinity of, and in the close-packed directions from, precipitates. The precipitate number density must therefore be higher than that of voids: $N_{\mathrm{ppt}} \geq N$. The parameter $\eta_{\mathrm{bar}}=\eta_{\mathrm{ppt}}$ is equal to the total cross-sections of precipitates averaged over all $2 n$ close-packed crystallographic directions from which the SIA clusters can arrive, and normalised to that for the random distribution of defects. If voids are smaller than precipitates, $r \leq r_{\text {ppt }}$, then

$$
\eta_{\mathrm{ppt}}=\frac{1}{2 n \sigma_{\mathrm{ppt}}}\left(2 n \pi r_{\mathrm{ppt}}^{2} N_{\mathrm{ppt}}-\pi r^{2} N\right) .
$$

The first term in brackets is the total cross-section of precipitates in all $2 n$ directions. The second term stands for the reduction of the precipitate cross-section by screening due to voids and is equal to the total cross-section of voids in one direction, because a void can shadow a precipitate from only one direction. In addition, large voids, $r>>r_{\text {ppt }}$, can block up to $n$, i.e. half, of all directions for the arrival of the SIA clusters. We introduce a function $\vartheta_{\mathrm{ppt}}(r): \vartheta_{\mathrm{ppt}}\left(r=r_{\mathrm{ppt}}\right)=1$ and $\lim _{r \rightarrow \infty} \vartheta_{\mathrm{ppt}}(r)=n$, for the description of this effect. Finally,

$$
\eta_{\mathrm{ppt}}= \begin{cases}1-\frac{1}{2 n} \frac{r^{2} N}{r_{\mathrm{ppt}}^{2} N_{\mathrm{ppt}}}, \quad r \leq r_{\mathrm{ppt}}, \\ 1-\frac{\vartheta_{\mathrm{ppt}}(r)}{2 n} \frac{N}{N_{\mathrm{ppt}}}, \quad r>r_{\mathrm{ppt}}\end{cases}
$$

The correlation-screening factor of voids is derived in the same way:

$$
\eta_{\mathrm{V}}= \begin{cases}1-\frac{\vartheta_{\mathrm{V}}\left(r_{\mathrm{ppt}}\right)}{2 n}, & r \leq r_{\mathrm{ppt}}, \\ 1-\frac{1}{2 n} \frac{r_{\mathrm{ppt}}^{2}}{r^{2}}, & r>r_{\mathrm{ppt}} .\end{cases}
$$

There are two differences between equations (20) and (21), however. First, the number densities of voids and precipitates do not enter equation (21) since each void is affected by one precipitate; hence both terms in the brackets of equation (19) are proportional to the void number density, that is $2 n \pi r^{2} N-\pi r_{\mathrm{ppt}}^{2} N$. Second, unlike voids, precipitates, especially large ones, may have long strain fields, which repel the SIA clusters. Hence, the function $\vartheta_{\mathrm{V}}\left(r_{\mathrm{ppt}}\right)$ may be different from unity even at $r>r_{\mathrm{ppt}}$, but we did not take this into account in equation (21). And what is more important, a void growing in the repulsive stress field can be shielded from the SIA clusters from all directions: 


$$
\lim _{r_{\mathrm{ppt}} \rightarrow \infty} \vartheta_{\mathrm{V}}\left(r_{\mathrm{ppt}}\right)=2 n
$$

This would create the possibility for voids to grow until their size exceeds the range of the stress field. This conclusion should remain valid for voids in the compression side of edge dislocations as well.

\subsection{Interpretation of the universal swelling rate observed in stainless steels}

Before concluding this section, we would like to point out that the present analysis provides for the first time an interpretation of the steady-state swelling rate of 1\%/(NRT dpa) observed in austenitic stainless steels of different compositions [27]. Note that the swelling rate described by equation (17) is maximum if voids are completely screened from the SIA clusters, i.e. when $\eta_{\mathrm{V}}=0$, and if the recombination of the SIA clusters with vacancies is negligible. If these conditions hold, then the swelling rate is

$$
\frac{d S}{d \phi^{\mathrm{NRT}}} \approx \varepsilon_{\text {surv }} \varepsilon_{\mathrm{i}}^{\mathrm{g}} \frac{4 \pi r N}{4 \pi r N+Z_{\mathrm{v}} \rho} \approx \frac{1}{2} \varepsilon_{\text {surv }} \varepsilon_{\mathrm{i}}^{\mathrm{g}} .
$$

Here we replaced the ratio of sink strengths by one half, since, typically $4 \pi r N \approx Z_{\mathrm{v}} \rho$ in the case considered. Let us now make some estimates. The survival fraction of defects in cascades can be estimated as $\varepsilon_{\text {surv }}=0.1$, which is the value of $\sim 0.2$ calculated by MD $[47,48]$ divided by two. The factor of two is an estimate for additional recombination of defects during the annealing stage of cascades. Then, the observed swelling rate of $1 \%$ /(NRT dpa) [27] can be explained if $\varepsilon_{\mathrm{i}}^{\mathrm{g}} \approx 0.2$, which is close to the results of MD simulations of cascades [48] and to the best fit value obtained in [37] to reproduce swelling in neutron-irradiated $\mathrm{Cu}$ at low irradiation dose. This analysis is also supported by calculations presented on figure 4, where the curve with full squares illustrates a possible scenario of void swelling in stainless steels, when spatial correlations develop with irradiation dose. Thus, the universal steady-state swelling rate observed in austenitic stainless steels can be interpreted as equal to the half of the production bias, i.e. half of the fraction of SIAs that survive inter-cascade recombination and are produced as 1-D mobile clusters. The independence of the swelling rate on steel composition can be explained by the independence of the cascade process on the composition: the final defect structure at the end of the cascade process is defined in the early stages of a cascade when the energies involved are still much higher than the differences in various binding energies of defects with solute atoms.

The correlation of the incubation period of swelling with the formation of the dislocation network observed [27] may be connected with an increase of the volume for the nucleation of voids, where voids are screened from the SIA clusters. Higher dislocation density also corresponds to smaller dislocation climb rate, which might be essential for preserving void-dislocation correlations. In this context it is worth mentioning that, at relatively high temperature, prior cold-work is observed to increase swelling rates both in pure iron and austenitic alloys, by accelerating the void nucleation and thereby shortening the duration of the transient period of swelling [74,75].

Summarising this section, we conclude that spatial correlations between voids and other defects must be an effective mechanism for void swelling, or, more exactly, for maintaining the operation of production bias at large void size. 


\section{INFLUENCE OF SOLUTE ATMOSPHERES}

The RIS of alloying elements was first observed by Okamoto et al. [15] near voids in a high-purity 18Cr-8Ni-1Si stainless steel during in-situ bombardment in a high-voltage electron microscope. It has been observed since in many other materials irradiated with different energetic particles near sinks of point defects such as voids, dislocations and grain boundaries. It is also responsible for the radiation-induced modification of phase diagrams of alloys [76,77]. It changes not only the stability of second-phase precipitates observed under thermal-equilibrium conditions but also their composition and, in some cases, leads to appearance of new phases. It also affects mechanical properties of alloys by changing chemical composition near dislocations and grain boundaries. In addition, it has been found to affect accumulation of radiation damage, for there is correlation between swelling rates and partial diffusion coefficients of impurity atoms [78,79]. Extensive observations of RIS and precipitation that precedes and accompanies void swelling led Garner [80] to a hypothesis that micro-chemical evolution is one of the major factors that controls the onset of swelling in stainless steels. Thus, RIS is a common phenomenon that influences microstructure evolution of structural materials under irradiation.

If the primary damage is in the form of Frenkel pairs, the main effect of RIS on damage accumulation is to change the absorption/emission rates of mobile point defects by different sinks [81-83]. The change of sink strength due to RIS is generally different for vacancies and single SIAs thus leading to appearance of an additional bias. Marwick [83] estimated theoretically that the RIS-induced bias factor of a free surface in a foil of Fe-Cr-Ni alloy is $\sim 10 \%$, which is of the order of, or even higher than, the dislocation bias (see discussion of the value of the latter at the end of Section 2). Thus, damage accumulation in irradiated alloys has to be different from that in pure metals.

Further development of these ideas in the framework of the SRT was undertaken by Golubov et al. $[84,85]$. The main difficulty encountered was the fact that RIS takes place near all sinks of point defects, and thus affects them in a similar way and produces similar changes in sink efficiencies ( $Z_{\mathrm{i}}$ and $Z_{\mathrm{v}}$ in equation (1)). Hence, at a first sight, the overall effect, which is proportional to the difference of sink efficiencies, $B$, must be small. It was recognised that this is only true if the influence of dislocation climb on RIS is neglected. So, according to the theory proposed, in an alloy, the swelling rate has the same functional form as in a pure metal but with an effective bias factor, $B^{\text {eff }}$, which, to a first approximation, is a sum of the bias factors due to the dislocation stress field, $B$, and the RIS near voids, $B_{\mathrm{V}}^{\mathrm{RIS}}$, and dislocations, $B_{\mathrm{d}}^{\mathrm{RIS}}$ :

$$
B^{\mathrm{eff}}=B+B_{\mathrm{d}}^{\mathrm{RIS}}-B_{\mathrm{V}}^{\mathrm{RIS}}
$$

In this equation, $B_{\mathrm{d}}^{\mathrm{RIS}}$ depends on the rate of dislocation climb, and hence on the swelling rate, $\dot{S}$, and $B_{\mathrm{V}}^{\mathrm{RIS}}$ of a small void is equal to $B_{\mathrm{d}}^{\mathrm{RIS}}$ of immobile dislocation, as shown in [85]:

$$
B_{\mathrm{d}}^{\mathrm{RIS}}(\dot{S} \rightarrow 0)=B_{\mathrm{V}}^{\mathrm{RIS}}
$$

Thus, if the dependence of the dislocation bias factor on the climb velocity is neglected, the effective bias would be equal to the dislocation bias factor. It was proposed in $[84,85]$ that, for the swelling rates observed, the segregation bias factor of dislocations should be negligible compared with that of voids: $\left|B_{\mathrm{d}}^{\mathrm{RIS}}\right|<<\left|B_{\mathrm{V}}^{\mathrm{RIS}}\right|$, since the RIS profiles at moving dislocations should be much flatter. 


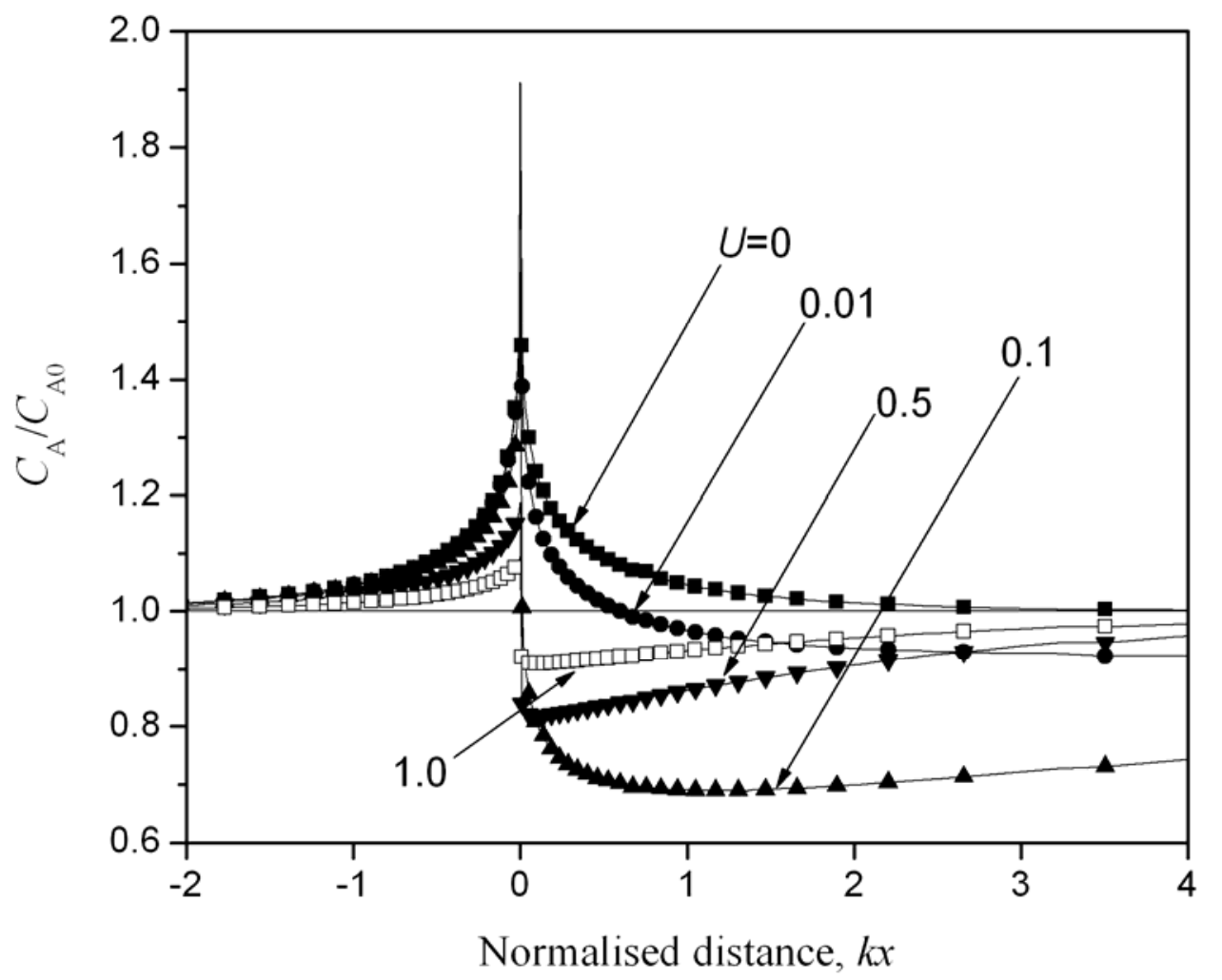

Figure 5. Concentration profiles of a component $\mathrm{A}$ in a binary $\mathrm{AB}$ alloy, $C_{\mathrm{A}}$, normalized by the initial concentration $C_{\mathrm{A} 0}$, calculated near a plain grain boundary for different velocities of the boundary motion. The boundary moves from the left to the right.

The conjecture about the absence of segregation profiles at moving dislocations is supported by the observations of the RIS profiles near moving grain boundaries (see e.g. [86]). A theoretical analysis of RIS profiles in a substitutional binary alloy of A and B components near a moving planar grain boundary was performed in [87] using the segregation model of Wiedersich et al. [88] in the framework of the SRT. Some results on the steady-state concentration profile of A component, $C_{\mathrm{A}}$, normalised by the initial concentration $C_{\mathrm{A} 0}$, calculated for different velocities of the boundary motion, $V$, are shown in figure 5. In this figure, the distance $x$ from the boundary is normalised by the mean distance between sinks for point defects, $k^{-1}$, where $k^{2}$ is the total sink strength for 3-D migrating point defects. Typically, $k^{2}=10^{14} \mathrm{~m}^{-2}$, so that $k x=1$ corresponds to $x=100 \mathrm{~nm}$. The velocity $V$ is normalised as $U=V k / 2 G$, where $G$ is the damage rate. As can be seen from the figure, the RIS profile depends significantly on the boundary velocity and disappears when $U \approx 1$.

For a moving dislocation or growing void, the analysis is more complicated. Some estimates can be made, however, by assuming that the results obtained for the moving grain boundary can be used for other sinks. The swelling rate depends on the velocity of edge dislocation climb, $V_{d}$, as

$$
\dot{S}=V_{\mathrm{d}} \rho b,
$$

where $b$ is the Burgers vector length of dislocations. Assuming $\rho=k^{2} / 2$, which is frequently the case, e.g. in austenitic stainless steels [89], one obtains for the normalised velocity of dislocation 


$$
U_{\mathrm{d}} \approx V_{\mathrm{d}} k / 2 G=\dot{S} / k b G
$$

For $k^{2}=10^{14} \mathrm{~m}^{-2}, \quad b \approx 0.3 \mathrm{~nm}$ and $\dot{S} / G \approx 1 \% / \mathrm{dpa}[27,89]$, the normalised velocity is $U_{\mathrm{d}} \approx 3$. According to figure 5 , the segregation for such a velocity must be very small. Similar analysis can be performed for voids. The swelling rate is connected with the velocity of the void surface movement, $V_{\mathrm{V}}=\dot{r}$, via the following equation

$$
\dot{S}=V_{\mathrm{V}} k_{\mathrm{V}}^{2} r
$$

where $k_{\mathrm{V}}^{2}=4 \pi r N$ is the sink strength of voids. Assuming $k_{\mathrm{V}}^{2}=k^{2} / 2$, one obtains

$$
U_{\mathrm{V}} \approx V_{\mathrm{V}} k / 2 G=\dot{S} / k r G
$$

It follows from equations (27) and (29) that $U_{\mathrm{V}} \approx U_{\mathrm{d}} b / r$, and so $U_{\mathrm{V}}<<U_{\mathrm{d}}$ for $r>b$. Hence, the effect of void surface motion on segregation profiles must be negligible.

Similar or even bigger effect of RIS on void growth may be expected in neutron irradiation. The migration properties of the SIA clusters are known to be sensitive to the presence of lattice imperfections and so the cluster interaction with lattice defects (voids, dislocations, etc.) must be strongly affected by enrichment/depletion by solute elements. If solute atmospheres develop near dislocations as well as near voids and both repel the SIA clusters, the result would be a decrease in the swelling rate due to enhanced recombination rate of SIA clusters with vacancies, which can be a strong effect. If, on the other hand, only voids are affected by RIS and repel the clusters, the effect would be similar to decrease of the correlation-screening factor, $\eta_{\mathrm{V}} \rightarrow 0$, due to shadowing effect. The spatial correlation and screening would then be due to dispersed solute atoms instead of precipitates or dislocations. In the limiting case of complete screening of voids distributed randomly over the volume, the swelling rate can be described by equation (17) with $\eta_{\mathrm{V}}=0, \eta_{\mathrm{ppt}}=1$ and the cross-section of repulsive barriers of precipitates and voids, $\sigma_{\mathrm{bar}}=\sigma_{\mathrm{ppt}}+\sigma_{\mathrm{V}}$ :

$$
\frac{d S}{d \phi}=\varepsilon_{\mathrm{i}}^{\mathrm{g}} p_{3 \mathrm{~V}} \frac{1+2 \sigma_{\mathrm{bar}} / \sigma_{\mathrm{d}}}{\left[1+\sigma_{\mathrm{bar}} / \sigma_{\mathrm{d}}\right]^{2}} .
$$

The irradiation doses required for the development of RIS profiles are known to be in the same range as for the incubation period of swelling, i.e. from about one to several tens of dpa. Thus, the dependence presented in figure 4 by full squares (see description in next paragraph after equation (18)) can also be used for schematic illustration of the effect of RIS on swelling rate. Finally, we conclude that the effect of RIS on swelling is similar to the screening effect from precipitates and dislocations and may even solely explain unlimited void growth in the framework of PBM. 


\section{SUMMARY AND OUTLOOK}

Research over more than half of a century has resulted in substantial understanding of the response of various materials to neutron irradiation. Theory has made a significant contribution to this but failed to acquire a leading role in creating radiation-resistant materials. This is despite the fact that, from early on, there was evidence that the mechanisms responsible for the microstructure evolution under neutron irradiation are qualitatively different from those during irradiation with $1 \mathrm{MeV}$ electrons. It was known, for example, that features of the initial damage, such as vacancy and interstitial clustering, were different, and that the large-scale behaviour of materials under neutron irradiation disobeys predictions of the SRT [28]. Their potential importance was largely ignored and so the SRT dominated theoretical interpretation of experimental data on neutron and heavy-ion irradiations, with dubious outcome.

The PBM gives the most promising theoretical approach, which explains several striking observations listed in the introduction section and its validity has been confirmed by carefully-planned experiments (e.g. [36]). The uniqueness of these time-consuming and expensive experiments in the history of radiation damage physics should be emphasised: they provided direct evidence that the driving force for damage accumulation under neutron irradiation is orders of magnitude stronger than under highenergy electron irradiation of similar NRT dpa rate.

In its present form, the PBM is valid for small-dose irradiation of pure materials but it does not fully incorporate the effects of spatial correlations, despite the fact that the main observations explained by the model are due to them, i.e. void lattice formation due to correlations between voids; and grain boundary and grain size effects due to correlation of voids with grain boundaries. This seems to be the main deficiency of the model that prevents its wider application.

Our analysis of the problem can be summarised in the following statements, which we propose for consideration and judgement by the reader.

1. Voids can grow indefinitely if the production bias, i.e. the fraction of SIAs produced in cascades in the form of 1-D mobile clusters, is smaller than a certain value of the order of the dislocation bias, but, according to our current understanding, this condition is unlikely to be satisfied for neutron irradiation. To elucidate the reasons behind the discrepancy between different approaches for estimating the dislocation bias, this area should be revisited.

2. Spatial correlations always occur under cascade damage conditions as a result of interactions of 1$\mathrm{D}$ migrating SIA clusters with defects, such as voids and dislocations. The void super-lattice is only one type of correlations, which is realised at high void density. The very absence of a void lattice must be an indication of the existence of correlation of voids with other defects. Densities of defects, such as voids, dislocations and precipitates, govern prevalence of particular types of spatial correlations. The development of correlations minimises the void-SIA cluster interaction intensity by screening voids from the SIA clusters. The characteristic range of spatial correlations is of the order of the mean-free path of 1-D migrating SIA clusters, which is typically at least an order of magnitude longer than the average distance between the defects, $\sim \mu \mathrm{m}$. The correlation effects are known to exist even if the point defects executing 3-D random walk are the only migrating entities (see figure 22 in [90]), but their effect on microstructure evolution is negligible. In contrast, under cascade irradiation, correlation effects are large and their existence becomes the only controlling factor for damage accumulation at high enough doses.

3. Voids that are not screened from the SIA clusters, i.e. randomly distributed over the volume, stop growing above a certain radius. Voids that are screened by precipitates having no long-range strain field stop growing at a higher void size. Precipitates with long-range strain fields repulsive for the SIA clusters and compressive sides of edge dislocations are two examples of regions where voids can grow until their size exceeds the range of the repulsive field. 
4. If repulsive barriers for the SIA clusters, e.g. precipitates and compressive regions of edge dislocations, are randomly distributed and not correlated with voids, they decrease the swelling rate but do not affect the void saturation radius.

5. The steady-state swelling rate of $\sim 1 \% /$ dpa observed in austenitic stainless steels can be interpreted as being equal to about half of the production bias, i.e. half of the fraction of SIAs that survive inter-cascade recombination and are produced as 1-D mobile clusters. Its weak dependence on steel composition is probably because the final defect structure is governed by early stages of the cascade, when the energies involved are much higher than differences in binding energies of defects with solute atoms.

6. The observed correlation of the incubation period of swelling with the formation of a dislocation network may be connected with an increase of the volume for the nucleation and growth of voids in which voids are screened from the SIA clusters. Higher dislocation density also corresponds to smaller dislocation climb rate, which might be essential for preserving void-dislocation correlations.

7. The atmospheres of solute elements near voids may repel SIA clusters and assist or even solely explain the unlimited void growth within the framework of PBM.

8. Solute, impurity and transmuted atoms may decrease the diffusion rate of SIA clusters, thus increasing the recombination rate of clusters with freely migrating vacancies. In the limiting case of very high binding energy of SIA clusters with impurity atoms, the so-called 'Singh-Foreman catastrophe' may occur, i.e. accumulation of an extremely high density of immobilised SIA clusters that prevent further evolution of the microstructure. This effect can explain the existence of the incubation period of swelling commonly observed in stainless steels. In this case, the end of the incubation period can be attributed to cleaning the matrix of solute atoms by segregation to sinks. It suggests that material resistance to irradiation can be improved by adding suitable solutes to cause the 'Singh-Foreman catastrophe' for a desired period of time. Another way is to destroy spatial correlations of voids with other defects by any means.

Thus, it is suggested to add two additional points to the list of distinguishing features of microstructure evolution under neutron compared to electron irradiation at high enough doses: transmutation of atoms, which transforms even pure metals to alloys, and development of spatial correlations. Generally, it follows from the analysis presented that the presence of 1-D mobile SIA clusters in crystals under cascade irradiation makes the development of spatial correlations between voids and other microstructural features inevitable. Hence, all theories developed to date are incomplete and incapable of accurately predicting damage accumulation in alloys under cascade irradiation at high doses. The development of a predictive theory requires revisiting all essential constituent elements of the theory. These include the nucleation, growth and movement of voids and other lattice defects in the presence of mutual spatial correlations, for all heterogeneous aspects of microstructure evolution deserve attention. Due to increased geometrical complexity of these problems compared to those without correlations, their solution will probably require much closer linkage between different approaches, such as quantum mechanical, MD, Monte Carlo and analytical, than is currently the case. Carefully planned experiments must be a central part of such studies. In addition, a revival of the RIS theory and its further development for accounting for SIA clusters is necessary for understanding the sensitivity of microstructure to material composition. Generally, the challenge is to create such a theory, where the mean-field approach in its conventional form is abandoned, a task not attempted before. 


\section{REFERENCES}

[1] M.J. Norgett, M.T. Robinson and I.M. Torrens, Nucl. Eng. and Design 3350 (1975).

[2] K.C. Russel, Acta Met. 19753 (1971).

[3] J.L. Katz and H. Wiedersich, J. Chem. Phys. 551414 (1971).

[4] S.D. Harkness and Che-Yu Lie, in Radiation Damage in Reactor Materials (I.A.E.A. Vienna) 2189 (1969).

[5] R. Bullough, B.L. Eyre and R.C. Perrin, J. Nucl. Appl. Technol. 9346 (1970).

[6] S.D. Harkness and Che-Yu Li, Met. Trans. 21457 (1971).

[7] H. Wiedersich, Rad. Eff. 12111 (1972).

[8] A.D. Brailsford and R. Bullough, J. Nucl. Mater. 4887 (1973).

[9] A.D. Brailsford and R. Bullough, Philos. Trans. Roy. Soc. A 30287 (1981).

[10] R. Bullough, B.L. Eyre and K. Krishan, Proc. R. Soc. Lond. A 34681 (1975).

[11] G.W. Greenwood, A.J.E. Foreman and D.E. Rimmer, J. Nucl. Mater. 4305 (1959).

[12] C. Cawthorne and E.J. Fulton, Symp. The Nature of Small Defect Clusters, M.J. Makin ed., vol. 2, AERE-R-5269, Harwell, p.446 (1966).

[13] C. Cawthorne and E.J. Fulton, Nature 216575 (1967).

[14] T.R. Anthony, U.S. Atomic Energy, CONF-710601, 630 (1972).

[15] P.R. Okamoto, S.D. Harkness and J.J. Laidler, ANS Trans. 1670 (1973).

[16] A.J.E. Foreman, UKAE Authority Harwell Report No. AERE-R-7135 (1972).

[17] J.H. Evans, Nature 229403 (1971).

[18] B.N. Singh and S.J. Zinkle, J. Nucl. Mater. 206212 (1993).

[19] B.N. Singh, Radiat. Eff. 148383 (1999).

[20] B.N. Singh, J.B. Bilde-SØrensen and T. Leffers, J. Nucl. Mater. 122-123 542 (1984).

[21] J.O. Stiegler and E.E. Bloom, Radiat. Eff. 833 (1971).

[22] R.E. Stoller and G.R. Odette, J. Nucl. Mater. 154286 (1988).

[23] B.N. Singh and T. Leffers, Vop. At. Nauki Tekh., Ser.: Fiz. Radiat. Povrezh. Radiat. Mater. 153 (1981).

[24] B.N. Singh, T. Leffers and A. Horsewell, Philos. Mag. A 53233 (1986).

[25] C.A. English, B.L. Eyre and W. Muncie, Philos. Mag. A 56453 (1987).

[26] C. Cawthorne, Proc. UK/US Fast Reactor Exchange Meeting on Cladding and Duct Materials, CONF-7910113, HEDL, Richland, October 30-November 2 (1979).

[27] F.A. Garner, M.B. Toloczko and B.H. Sencer, J. Nucl. Mater. 276123 (2000).

[28] E.A. Little and D.A. Stow, J. Nucl. Mater. 8725 (1979).

[29] H. Matsui, D.S. Gelles and Y. Kohno, in Effects of Radiation on Materials, ASTM STP 1125928 (1992).

[30] C.H. Woo and B.N. Singh, Phys. Stat. Sol. (b) 159609 (1990).

[31] C.H. Woo and B.N. Singh, Philos. Mag. A 65889 (1992).

[32] B.N. Singh and A.J.E. Foreman, Philos. Mag. A 66975 (1992).

[33] H. Trinkaus, B.N. Singh and A.J.E. Foreman, J. Nucl. Mater. 1991 (1992).

[34] B.N. Singh, S.I. Golubov, H. Trinkaus, et al., J. Nucl. Mater. 251107 (1997).

[35] B.N. Singh, M. Eldrup, S.J. Zinkle, et al., Philos. Mag. A 821137 (2002).

[36] B.N. Singh, M. Eldrup, A. Horsewell, et al., Philos. Mag. A 802629 (2000).

[37] S.I. Golubov, B.N. Singh and H. Trinkaus, Philos. Mag. A 812533 (2001).

[38] D. Terentyev, L. Malerba and A.V. Barashev, Philos. Mag. Lett. 85587 (2005).

[39] A.C. Arokiam, A.V. Barashev, D.J. Bacon, et al., Philos. Mag. 87925 (2007).

[40] B. von Guerard and J. Peisl, in: Proc. Int. Conf. On the Fundamental Aspects of Radiation Damage in Metals, Gatlinburg, TN, eds. M. Robertson and W. Young, CONF-751006, USERDA, p.287, 1975.

[41] B. von Guerard and J. Peisl, J. Appl. Cryst. 8161 (1975).

[42] B. von Guerard, D. Grasse and J. Peisl, Phys. Rev. Lett. 44262 (1980).

[43] D. Grasse, B. von Guerard and J. Peisl, J. Nucl. Mater. 120304 (1984). 
[44] R. Rauch, J. Peisl, A. Schmalzbauer, et al., J. Nucl. Mater. 168101 (1989).

[45] T. Diaz de la Rubia and M.W. Guinan, Phys. Rev. Lett. 662766 (1991).

[46] A.J.E. Foreman, W.J. Phythian and C.A. English, Philos. Mag. A 66671 (1992).

[47] D.J. Bacon, A.F. Calder, F. Gao, et al., Nucl. Instr. Meth. B 10237 (1995).

[48] D.J. Bacon, Yu.N. Osetsky, R. Stoller, et al., J. Nucl. Mater. 323152 (2003).

[49] Yu.N. Osetsky, D.J. Bacon, A. Serra, et al., Philos. Mag. A 8361 (2003).

[50] M. Kiritani, J. Nucl. Mater. 251237 (1997).

[51] T. Hayashi, K. Fukmuto and H. Matsui, J. Nucl. Mater. 307-311 993 (2002).

[52] K. Arakawa, K. Ono, M. Isshiki, et al., Science 318956 (2007).

[53] A.H. Cottrell, Report on Strength of Solids, Physical Society, London 30 (1948).

[54] J.D. Eshelby, Solid St. Phys. 379 (1956).

[55] P.T. Heald and M.V. Speight, Acta Metall. 231389 (1975).

[56] K.M. Miller, J. Nucl. Mater. 84167 (1979).

[57] G.P. Walters, J. Nucl. Mater. 136263 (1985).

[58] W. Jäger and H. Trinkaus, J. Nucl. Mater. 205394 (1993).

[59] A.V. Barashev and S.I. Golubov, submitted to Philos. Mag. (2008).

[60] D. Helbing and T. Vicsek, New Journal of Physics 113.1 (1999).

[61] A.V. Barashev, S.I. Golubov and H. Trinkaus, Philos. Mag. A 812515 (2001).

[62] K. Farrell and J.T. Houston, J. Nucl. Mater. 35352 (1970).

[63] K. Krishan, B.N. Singh and T. Leffers J. Nucl. Mater. 133-134 439 (1985).

[64] L.K. Mansur, M.R. Hayns and E.H. Lee, Proc. Conf. Phase Stability under Irradiation, AIME, Warrendale, PA, p.359 (1981).

[65] A.F. Rowcliffe and E.H. Lee, J. Nucl. Mater. 108-109 306 (1982).

[66] P.J. Maziasz, J. Nucl. Mater. 122-123 472 (1984).

[67] D.F. Pedraza and P.J. Maziasz, in Radiation Induced Changes in Microstructure, ASTM STP 955 161 (1987).

[68] R.M. Boothby and T.M. Williams, J. Nucl. Mater. 152123 (1988).

[69] A. Risbet, G. Brebec, J.M. Lanore, et al., J. Nucl. Mater. 56348 (1975).

[70] J.M. Lanore, L. Glowinski, A. Risbet, et al., in Proc. Int. Conf. On the Fundamental Aspects of Radiation Damage in Metals, Gatlinburg, TN, eds. M. Robertson and W. Young, CONF-751006, USERDA, p.1169 (1975).

[71] A.V. Kozlov, I.A. Portnykh, L.A. Skryabin, et al., J. Nucl. Mater. 307-311 956 (2002).

[72] I.A. Portnykh, A.V. Kozlov, V.L. Panchenko, et al., J. Nucl. Mater. 367-370 925 (2007).

[73] Q. Xu, T. Yoshiie and K. Sato, Philos. Mag. Lett. 88353 (2008).

[74] F.A. Garner, C.A. Black and D.J. Edwards, J. Nucl. Mater. 245124 (1997).

[75] A.M. Dvoriashin, S.I. Porollo, Yu.V. Konobeev, et al., J. Nucl. Mater. 283-287 157 (2000).

[76] A.D. Marwick, Nucl. Instrum. Meth. 182/183 827 (1981).

[77] K.S. Russel, Prog. in Mater. Sci. 28229 (1984).

[78] H. Venker and K. Ehrlich, J. Nucl. Mater. 60347 (1976).

[79] H. Venker, P. Giesecke and K. Ehrlich, in Radiation Effects in Breeder Reactor Structural Materials. (Proc. Inter. Conf., Scottsdale, Arizona, 19-23 June 1977), p.415 (1977).

[80] F.A. Garner, in Phase Stability During Irradiation, J.R. Holland, L.K. Mansur, D.I. Potter, eds. (Met. Soc. AIME, 1981) p.165.

[81] A.D. Brailsford, J. Nucl. Mater. 567 (1975).

[82] W.G. Wolfer, J. Nucl. Mater. 114292 (1983).

[83] A.D. Marwick, J. Nucl. Mater. 13568 (1985).

[84] S.I. Golubov, Metallofizika 1110 (1989) (in Russian).

[85] S.I. Golubov, V.P. Ginkin and A.M. Strokova, Mater. Sci. Forum 97-99 97 (1992).

[86] S.M. Bruemmer, L.A. Charlot, J.S. Vetrano, et al., in Microstructure of irradiated materials (Symp. Y, Nov. 29 - Dec. 1 1994, Boston, Massachusetts, USA), eds. I.M. Robertson et al., Pittsburg. 373119 (1995).

[87] A.V. Barashev and S.I. Golubov, Preprint IPPE 2518, Obninsk (1996) (in Russian). 
[88] H. Wiedersich, P.R. Okamoto and N.Q. Lam, J. Nucl. Mater. 8398 (1979).

[89] F.A. Garner, J. Nucl. Mater. 20598 (1993).

[90] U.M. Gösele, Prog. Reaction Kinetics 1363 (1984). 
ORNL/TM-2008/141

\section{INTERNAL DISTRIBUTION}

1. S. J. Zinkle

2. R. K. Nanstad

3. R. E. Stoller

4. M. Stocks

5. Yu.N. Osetsky

6. J. R. Morris

7. D. N. Nicholson

8. D. Stewart

9. J. T. Busby

10. L. K. Mansur

11. M. K. Miller

12. ORNL office of Technical Information

\section{EXTERNAL DISTRIBUTION}

13-18. Pacific Northwest National Laboratory, Structural Materials Research, P.O. Box 999,

Richland, WA 99352, USA, frank.garner@pnl.gov, hl.heinisch@pnl.gov, rj.kurtz@pnl.gov,

Fei.Gao@pnl.gov, dan.edwards@pnl.gov, david.gelles@pnl.gov
F.A. Garner
H. L. Heinisch
R.J. Kurtz
F. Gao
D.J. Edwards
D.S. Gelles

19. University of Michigan, Nuclear Engineering and Radiological Sciences, Cooley Building, Ann Ardor, MI 48109-2104, USA, gsw@umich.edu

G.S. Was

20. University of California, Department of Chemical and Nuclear Engineering, Ward Memorial Drive, Santa Barbara, CA 93106, USA, odette@engineering.ucsb.edu

G.R. Odette

21. University of California, Department of Nuclear Engineering, Berkeley, CA 94720-1730,

USA, bdwirth@nuc.berkeley.edu

B.D. Wirth

22-23. University of California, Department of Mechanical and Aerospace Engineering, Los Angeles, CA 90095, USA, nghoniem@gmail.com, sharams@gmail.com

N.M. Ghoniem

S. Sharafat 
24. University of Wisconsin-Madison, COLLEGE OF ENGINEERING, ENGINEERING PHYSICS, 529 Engineering Research Building, 1500 Engineering Dr, Madison, WI 53706, allen@engr.wisc.edu

T.R. Allen

25. Argonne National Laboratory, Materials Science Division, Argonne, Illinois 60439, USA, kirk@anl.gov

M.A. Kirk

26 Lawrence Livermore National Laboratory, Livermore, CA 94550, USA, wolferl@llnl.gov W.G. Wolfer

27-29. University of Illinois, Urbana-Champaign, USA, jstubbins@express.cities.uiuc.edu, averback@uiuc.edu, bellon@illinois.edu

J. Stubbins

R. Averback

P. Bellon

30. Department of Materials Science and Engineering, Room 13-5050, 77 Massachusetts Avenue, Cambridge, MA 02139, USA, kenruss@mit.edu

K.C. Russell

31. Department of Chemical and Biomolecular Engineering, GWC Whiting School of Engineering, The Johns Hopkins University, 221 Maryland Hall, 3400 North Charles Street, Baltimore, MD 21218, USA, jlk@jhu.edu

J.L. Katz

32. The University of Liverpool, Department of Engineering, Brownlow Hill, Liverpool, L69 3GH, UK, A.Barashev@liverpool.ac.uk, djbacon@liverpool.ac.uk

D.J. Bacon

33.4 Long Meadow, Manor Road, Goring, Reading RG8 9EG, UK, r.bullough@btinternet.com R. Bullough

34-36. University of Oxford, Department of Materials, Parks Rd., Oxford OX1 3PH, UK, mike.jenkins@materials.ox.ac.uk, steve.roberts@materials.ox.ac.uk, john.titchmarsh@materials.ox.ac.uk

M.L. Jenkins

S.G. Roberts

J. Titchmarsh

37. Nexia Solutions, UK, colin.english@materials.ox.ac.uk, colin.a.english@nexiasolutions.com C.A. English

38. EURATOM/UKAEA Fusion Association, Culham Science Centre, Oxfordshire OX14 3DB, UK, Sergei.Dudarev@UKAEA.org.uk

S.L. Dudarev

39-40. Risø National Laboratory, Materials Department, DK-4000 Roskilde, Denmark, bachu.singh@risoe.dk, morten.eldrup@risoe.dk

B.N. Singh

M. Eldrup 
41. Institut für Festkörperforschung des Forschungszentrums Jülich, W-5170 Jülich, Germany, susanne.trinkaus@gmx.de

H. Trinkaus

42. Universitat Politècnica de Catalunya, Dept. Matemàtica Aplicada III, ETSECCPB and CRNE, 08034, Barcelona, Spain, a.serra@upc.edu

A. Serra

43-45. State Scientific Center of Russian Federation, The Institute of Physics and Power Engineering, 249020 Obninsk, Russian Federation, konobeev@ippe.ru, vap@ippe.obninsk.ru,

ryabov@obninsk.com,

Yu.V. Konobeev

V.A. Pechenkin

V.A. Ryabov

46. Research and Development Institute of Power Engineering, Sverdlovsk Branch, P.O. Box 29, Zarechny, Sverdlovsk region 624250, Russian Federation, alextim@e-sky.ru

A.V. Kozlov

47-48. RRC Kurchatov Institute, Kurchatov Sq.1, 123182, Moscow, Russian Federation, Vladimir.Borodin@imf.fzk.de, ryazanoff@comail.ru

V.A. Borodin

A.I. Ryazanov

49-50. SCK-CEN, RMO Department, Boeretang 200, B-2400 Mol, Belgium, lmalerba@sckcen.be, dterenty@sckcen.be

L. Malerba

D. Terentiev

51. LMPGM, UMR CNRS 8517, USTL and ENSCL, F-59655 Villeneuve d'Ascq Cedex, France, charlotte.becquart@univ-lille1.fr

C.S. Becquart

52. EDF-R\&D Department MMC, Les Renardieres, F-77818 Moret sur Loint Cedex, France, christophe.domain@edf.fr

C. Domain

53. Department of Materials and Mechanics of Components, EDF-R\&D, Les Renardières, F-77250 Moret-sur-Loing, France, olsson.par@gmail.com

P. Olsson

54. Service de Recherches de Metallurgie Physique, DMN-SRMP, CEA Saclay, 91191 Gif-surYvette, France, Frederic.soisson@cea.fr

F. Soisson

55. Research Reactor Institute, Kyoto University, Osaka 590-0494, Japan, xu@rri.kyoto-u.ac.jp Q. Xu 
56-57. Institute for Material Research, Tohoku University, Katahira 2-1-1, Aoba-ku, Sendai 980-8577, Japan, matsui@imr.edu, hasegawa@imr.tohoku.ac.jp

H. Matsui

M. Hasegawa

58. Research Center for Ultra-High Voltage Electron Microscopy, Osaka University, 7-1, Mihogaoka, Ibaraki, Osaka 567-0047, Japan, arakawak@uhvem.osaka-u.ac.jp

K. Arakawa

59. Research Institute for Applied Mechanics, Kyushu University, 6-1 Kasuga-koen, Kasuga, Fukuoka 816-8580, Japan, kuramoto@himiko.riam.kyushu-u.ac.jp

E. Kuramoto

60. The University of Tokyo, ishino@k9.dion.ne.jp

S. Ishino

61. Director of the Centre for Nuclear Energy Technology (CEKERT),

Sweden,janne@neutron.kth.se

J. Wallenius

62. SC Kharkov Institute of Physics and Technology NAS of Ukraine, Akademicheskaya Str.1, Kharkov 61108, Ukraine, vdubinko@mail.ru

V.I. Dubinko

63. University of Helsinki, Finland, kai.nordlund@helsinki.fi

K. Nordlund

64-65. Imperial College London, Department of Materials, 105 Royal School of Mines, South Kensington Campus, m.finnis@imperial.ac.uk, a.sutton@imperial.ac.uk

M.W. Finnis

A. Sutton 